REPRESENTATION THEORY

An Electronic Journal of the American Mathematical Society

Volume 15, Pages 347-369 (April 20, 2011)

S 1088-4165(2011)00399-X

\title{
INTEGRAL HOMOLOGY OF LOOP GROUPS VIA LANGLANDS DUAL GROUPS
}

\author{
ZHIWEI YUN AND XINWEN ZHU
}

\begin{abstract}
Let $K$ be a connected compact Lie group, and $G$ its complexification. The homology of the based loop group $\Omega K$ with integer coefficients is naturally a $\mathbb{Z}$-Hopf algebra. After possibly inverting 2 or 3 , we identify $H_{*}(\Omega K, \mathbb{Z})$ with the Hopf algebra of algebraic functions on $B_{e}^{\vee}$, where $B^{\vee}$ is a Borel subgroup of the Langlands dual group scheme $G^{\vee}$ of $G$ and $B_{e}^{\vee}$ is the centralizer in $B^{\vee}$ of a regular nilpotent element $e \in \operatorname{Lie} B^{\vee}$. We also give a similar interpretation for the equivariant homology of $\Omega K$ under the maximal torus action.
\end{abstract}

\section{INTRODUCTION}

Let $K$ be a connected compact Lie group and $G$ its complexification. Let $\Omega K$ be the based loop space of $K$. Then the homology $H_{*}(\Omega K, \mathbb{Z})$ is a Hopf algebra over $\mathbb{Z}$. The goal of this paper is to describe this Hopf algebra canonically in terms of the Langlands dual group of $G$.

The rational (co)homology of $\Omega K$ is easy since rationally $K$ is the product of spheres. However, the integral (co)homology is much more subtle: $H^{*}(\Omega K, \mathbb{Z})$ is not a finitely generated $\mathbb{Z}$-algebra, and the simplest example $H^{*}(\Omega \mathrm{SU}(2), \mathbb{Z})$ involves divided power structures. The integral (co)homology of $\Omega K$ has been studied extensively by different methods.

The first method was pioneered by Bott (cf. B58]). He developed an algorithm, which theoretically determines the Hopf algebra structure of integral (co)homology of all based loop groups. By applying this algorithm, he gave explicit descriptions of the (co)homology of special unitary groups, orthogonal groups, and the exceptional group $G_{2}$. However, Bott's description depends on the choice of a "generating circle" as defined in loc. cit. and is therefore not canonical.

The second method to study the (co)homology of based loop groups comes from the theory of Kac-Moody groups. Namely, let $G$ be the complexification of $K$. This is a reductive algebraic group over $\mathbb{C}$. Let $F=\mathbb{C}((t))$ and $\mathcal{O}=\mathbb{C}[[t]]$. Then the quotient $\mathcal{G} r_{G}=G(F) / G(\mathcal{O})$, known as the affine Grassmannian of $G$, is a maximal partial flag variety of the Kac-Moody group $\widehat{G(F)}$ (a central extension of $G(F)$ ). Let $\Omega_{\text {pol }} K$ be the space of polynomial maps $\left(S^{1}, 1\right) \rightarrow\left(K, 1_{K}\right)$. More precisely, let us parametrize $S^{1}$ by $e^{i \phi}, \phi \in \mathbb{R}$, and let $K \subset S O(n, \mathbb{R})$ be an embedding. Then $\Omega_{\text {pol }} K$ is the space of maps from $\left(S^{1}, 1\right) \rightarrow\left(K, 1_{K}\right)$ such that when composed with $K \subset S O(n, \mathbb{R})$, the matrix entries of the maps are given by Laurent polynomials of

Received by the editors September 29, 2009 and, in revised form, October 24, 2010.

2010 Mathematics Subject Classification. Primary 57T10, 20 G07.

(C)2011 American Mathematical Society Reverts to public domain 28 years from publication 
$e^{i \phi}$. It is known that $\Omega_{\mathrm{pol}} K$ is homotopic to $\Omega K$. On the other hand, the obvious map

$$
\iota: \Omega_{\mathrm{pol}} K \rightarrow G(F) \rightarrow \mathcal{G} r_{G}
$$

is a homeomorphism between $\Omega_{\mathrm{pol}} K$ and $\mathcal{G} r_{G}$, which is $K$-equivariant (the action of $K$ on $\Omega_{\mathrm{pol}} K$ is the pointwise conjugation and on $\mathcal{G} r_{G}$ is the left multiplication). See [PS86] for details about these facts. Therefore, $\mathcal{G} r_{G}$ and $\Omega K$ have the same (co)homology groups. Kostant and Kumar (cf. KK86]) studied the topology of the (partial) flag varieties of arbitrary Kac-Moody groups. In particular, they determined the (equivariant) cohomology rings of these partial flag varieties, using an algebraic construction called the nil-Hecke ring. D. Peterson (unpublished work) realized that the nil-Hecke ring can also be used to study the homology ring of the affine Grassmannian. The affine variety $\operatorname{Spec} H_{*}\left(\mathcal{G} r_{G}\right)$ is usually called the Peterson variety.

In this paper, we will proceed from yet another perspective of this story, which is pioneered by Ginzburg (cf. G95]). We first recall the geometric Satake isomorphism developed by Lusztig ([L81), Ginzburg (G95), and Mirković and Vilonen (MV07]). We refer the details to the paper [MV07. Recall that $\mathcal{G} r_{G}$ is a union of projective varieties. Let $\mathcal{P}_{k}$ be the abelian category of $G(\mathcal{O})$-equivariant perverse sheaves with $k$-coefficients on $\mathcal{G} r_{G}$ whose supports are finite dimensional, where $k$ is some commutative noetherian ring of finite global dimension. The convolution product makes $\mathcal{P}_{k}$ into a tensor category, equipped with the functor $H^{*}\left(\mathcal{G} r_{G},-\right)$ of taking (hyper)cohomology as a fiber functor. The geometric Satake isomorphism claims that $H^{*}\left(\mathcal{G} r_{G},-\right)$ gives an equivalence of tensor categories

$$
H^{*}\left(\mathcal{G} r_{G},-\right): \mathcal{P}_{k} \cong \operatorname{Rep}\left(G_{k}^{\vee}, k\right),
$$

where $G^{\vee}$ is the Langlands dual group scheme of $G$, defined over $\mathbb{Z}$. Ginzburg observed that taking cohomology also gives rise to a tensor functor

$$
H^{*}\left(\mathcal{G} r_{G},-\right): \mathcal{P}_{k} \rightarrow \operatorname{Mod}\left(H^{*}\left(\mathcal{G} r_{G}, k\right)\right) .
$$

Assume that $G$ is simple and simply-connected and $k=\mathbb{C}$. By Tannakian formalism, one obtains a map of Hopf algebras

$$
H^{*}\left(\mathcal{G} r_{G}, \mathbb{C}\right) \rightarrow U\left(\mathfrak{g}_{\mathbb{C}}^{\vee}\right)
$$

where $\mathfrak{g}_{\mathbb{C}}^{\vee}$ is the Lie algebra of $G_{\mathbb{C}}^{\vee}$. Ginzburg proved that this map is injective, and the first Chern class $c_{1}\left(\mathcal{L}_{\text {det }}\right)$ of the determinant line bundle $\mathcal{L}_{\text {det }}$ on $\mathcal{G} r_{G}$ gets mapped to a regular nilpotent element $e$ in $\mathfrak{g}_{\mathbb{C}}^{\vee}$ under (1.2). Hence the map (1.2) factors through $U\left(\mathfrak{g}_{\mathbb{C}, e}^{\vee}\right) \subset U\left(\mathfrak{g}_{\mathbb{C}}^{\vee}\right)$, where $\mathfrak{g}_{\mathbb{C}, e}^{\vee}$ is the centralizer of $e$ in $\mathfrak{g}_{\mathbb{C}, e}^{\vee}$. Ginzburg concluded that this gives an isomorphism

$$
H^{*}\left(\mathcal{G} r_{G}, \mathbb{C}\right) \cong U\left(\mathfrak{g}_{\mathbb{C}, e}^{\vee}\right) .
$$

This paper is an extension of the above result to the (co)homology of $\mathcal{G} r_{G}$ with integer coefficients. According to MV07, the geometric Satake isomorphism holds for $k=\mathbb{Z}$. Therefore, it is natural to expect that (1.3) should hold over $\mathbb{Z}$ (hence should hold modulo every prime $p$ ). However, this is not the case, and it fails for two reasons:

First, as we already remarked, the integral cohomology ring $H^{*}\left(\mathcal{G} r_{G}, \mathbb{Z}\right)$ is not finitely generated over $\mathbb{Z}$ whereas $U\left(\mathfrak{g}_{\mathbb{Z}, e}^{\vee}\right)$ is. For this reason, we prefer to work with homology because it is a finitely generated $\mathbb{Z}$-algebra (under the Pontryagin 
product) by a result of Bott in [B58. The Tannakian formalism (cf. 93 ) gives us a natural homomorphism of group schemes

$$
\operatorname{Spec} H_{*}\left(\mathcal{G} r_{G}, \mathbb{Z}\right) \rightarrow G_{\mathbb{Z}}^{\vee}
$$

Second, although the first Chern class $c_{1}\left(\mathcal{L}_{\text {det }}\right)$ still gives rise to an element $e \in \mathfrak{g}_{\mathbb{Z}}^{\vee}$, it is not a regular nilpotent element modulo every prime $p$. When $e \bmod p$ is not regular, $U\left(\mathfrak{g}_{e}^{\vee}\right) \otimes \mathbb{F}_{p}$ is "too large" to be isomorphic to $H^{*}\left(\mathcal{G} r_{G}, \mathbb{F}_{p}\right)$. We must carefully exclude these $p$ 's.

Now the main theorem of this note reads as

1.1. Theorem (See Theorem 6.1(1)). Let $G$ be a reductive connected group over $\mathbb{C}$ such that its derived group $G^{\text {der }}$ is almost simple. Let $K$ be a maximal compact subgroup of $G$. Let $\ell_{G}$ be the square of the ratio of the lengths of long roots and the short roots of $G$ (so $\ell_{G}=1,2$ or 3$)$. Then there is a canonical isomorphism of group schemes over $\mathbb{Z}\left[1 / \ell_{G}\right]$ :

$$
\operatorname{Spec} H_{*}\left(\Omega K, \mathbb{Z}\left[1 / \ell_{G}\right]\right) \stackrel{\sim}{\rightarrow} B_{e}^{\vee}\left[1 / \ell_{G}\right],
$$

where $B^{\vee}$ is a fixed Borel subgroup of $G^{\vee}, e \in \operatorname{Lie} B^{\vee}$ is a regular nilpotent element given by (5.3), and $B_{e}^{\vee}$ is the centralizer of e in $B^{\vee}$.

This theorem describes the Hopf algebra structure of $H_{*}(\Omega K)$ in terms of a purely algebraic object $B_{e}^{\bigvee 1}$, at least after inverting $\ell_{G}$. In fact, one can also see the grading of $H_{*}(\Omega K)$ in terms of $B_{e}^{\vee}$ : the grading on $H_{*}(\Omega K)$ is the same thing as a $\mathbb{G}_{m}$-action on Spec $H_{*}(\Omega K)$, and this $\mathbb{G}_{m}$ action can be identified with the $\mathbb{G}_{m}$ action on $B_{e}^{\vee}$ given by the adjoint action through the cocharcter $2 \rho: \mathbb{G}_{m} \rightarrow T^{\vee}$.

We will also prove an equivariant version of the above theorem. Let $T$ be a maximal torus of $G$ such that $T \cap K$ is a maximal torus of $K$. Then $T \cap K$ acts on $\Omega K$ via conjugation. Let $R_{T}=H_{T \cap K}^{*}(\mathrm{pt}, \mathbb{Z})$. The $T \cap K$-equivariant homology $H_{*}^{T \cap K}(\Omega K, \mathbb{Z})$ (for precise definition, see (2.10) ) is an $R_{T}$-Hopf algebra, hence Spec $H_{*}^{T \cap K}(\Omega K, \mathbb{Z})$ is a group scheme over $R_{T}$. On the other hand, the $T$ equivariant Chern classes of line bundles on $\mathcal{G} r_{G}$ give an element $e^{T} \in \mathfrak{g}_{\mathbb{Z}}^{\vee} \otimes R_{T}\left[1 / n_{G}\right]$ (where $n_{G}$ is an integer explicitly given in Remark 5.8). The equivariant version of the main theorem reads as

1.2. Theorem (See Theorem 6.1(2)). Notations are the same as in Theorem 1.1. There is a canonical isomorphism of group schemes over $R_{T}\left[\frac{1}{\ell_{G} n_{G}}\right]$ :

$$
\operatorname{Spec} H_{*}^{T \cap K}\left(\Omega K, \mathbb{Z}\left[\frac{1}{\ell_{G} n_{G}}\right]\right) \stackrel{\sim}{\rightarrow} B_{e^{T}}^{\vee}\left[1 / \ell_{G}\right],
$$

where $B_{e^{T}}^{\vee}$ is the centralizer of $e^{T}$ in $B^{\vee} \times \operatorname{Spec} R_{T}\left[1 / n_{G}\right]$.

Finally, we also have descriptions of the cohomology $H^{*}(\Omega K)$ and the $K$-equivariant homology $H_{*}^{K}(\Omega K)$ in terms of group-theoretic data of $G^{\vee}$. For details, see $\oint 6.3$ and 86.5 .

Plan of the paper. In $\ 2$ we prove that the equivariant cohomology functor $H_{T}^{*}$ is a fiber functor from $\mathcal{P}$ to $R_{T}:=H_{T}^{*}(\mathrm{pt})$-modules that is canonically isomorphic to $H^{*} \otimes R_{T}$. We also describe how the cup product by $H^{*}\left(\mathcal{G} r_{G}\right)$ interacts with the tensor structure of the functor $H_{T}^{*}$. In $\S 3$, we construct a canonical homomorphism of groups schemes over $\mathbb{Z}$ from $\operatorname{Spec} H_{*}^{T}\left(\mathcal{G} r_{G}\right)$ to $B_{\mathbb{Z}}^{\vee}$. In $\oiint_{4}$ we digress to discussing

\footnotetext{
${ }^{1} \mathrm{~S}$. Kumar informed us that D. Peterson also obtained a similar result (unpublished).
} 
line bundles on the affine Grassmannian and their equivariant structures. In 95 , we determine the equivariant Chern class $c_{1}^{T}\left(\mathcal{L}_{\text {det }}\right)$ explicitly as an element in $\mathfrak{g}_{\mathbb{Z}}^{\vee} \otimes R_{T}$. Finally, in $\sqrt[6]{6}$, we prove our main theorem.

Convention and Notation. In this paper, we always assume that $G$ is a connected reductive algebraic group over $\mathbb{C}$. From \&4 we will assume that $G^{\text {der }}$ is almost simple. We will fix a Borel subgroup $B \subset G$ and a maximal torus $T \subset B$. Let $U$ be the unipotent radical of $B$. Let $\mathbb{X} \bullet(T)$ and $\mathbb{X}_{\bullet}(T)$ be the character group and cocharacher group of $T$. Let $K$ be the maximal compact subgroup of $G$ containing the maximal compact torus in $T$. We shall identify $\Omega K$ with $\Omega_{\text {pol }} K$, and denote them simply by $\Omega K$.

The Langlands dual group $G^{\vee}$ is the Chevalley group scheme over $\mathbb{Z}$ whose root system is identified with the coroot system of $G$. Later a Borel subgroup $B^{\vee} \subset G^{\vee}$ and a maximal torus $T^{\vee} \subset G^{\vee}$ will be fixed by the geometric Satake isomorphism. Let $U^{\vee}$ be the unipotent radical of $B^{\vee}$. The Lie algebras of these group schemes will be denoted by $\mathfrak{g}^{\vee}, \mathfrak{b}^{\vee}, \mathfrak{t}^{\vee}$ and $\mathfrak{u}^{\vee}$, which are free $\mathbb{Z}$-modules.

With dual groups in mind, our notation concerning the root system of $G$ is opposite to the usual one. Let $\Phi^{\vee}, \Phi$ be the set of roots and coroots of $G$. Coweights and coroots of $G$ will be denoted by $\lambda, \mu, \ldots$ and $\alpha, \beta, \ldots$, while weights and roots are denoted by $\lambda^{\vee}, \mu^{\vee}, \ldots$ and $\alpha^{\vee}, \beta^{\vee}, \ldots$ Let $2 \rho^{\vee}$ denote the sum of positive roots. We will give $\mathbb{X}_{\bullet}(T)$ a partial order such that $\lambda \leq \mu$ if and only if $\mu-\lambda$ is a positive integral combination of the simple coroots. Let $(-,-)_{\text {Kil }}$ be the Killing form on $\mathbb{X} .(T)$ given by:

$$
(x, y)_{\mathrm{Kil}}=\sum_{\alpha^{\vee} \in \Phi^{\vee}}\left\langle\alpha^{\vee}, x\right\rangle\left\langle\alpha^{\vee}, y\right\rangle .
$$

The (co)homology groups $H^{*}(-), H_{*}(-)$ are taken with $\mathbb{Z}$-coefficients unless otherwise specified. All tensor products $\otimes$ with no base ring specified are understood to be taken over $\mathbb{Z}$. For simplicity, we will write $\mathcal{P}$ for $\mathcal{P}_{\mathbb{Z}}$. For objects $\mathcal{F} \in \mathcal{P}$, we will abbreviate $H^{*}\left(\mathcal{G} r_{G}, \mathcal{F}\right), H_{T}^{*}\left(\mathcal{G} r_{G}, \mathcal{F}\right)$ by $H^{*}(\mathcal{F})$ and $H_{T}^{*}(\mathcal{F})$.

\section{EQUiVARIANT COHOMOLOGY FUNCTOR}

Let $G$ be a connected reductive algebraic group over $\mathbb{C}$. Let $F=\mathbb{C}((t))$ and $\mathcal{O}=\mathbb{C}[[t]]$. Let $\mathcal{G} r_{G}=G(F) / G(\mathcal{O})$ be the affine Grassmannian of $G$. Each coweight $\lambda \in \mathbb{X}_{\bullet}(T)$ determines a point $t^{\lambda} \in T(F)$, and hence a point in $\mathcal{G} r_{G}$, which we still denote by $t^{\lambda}$. For $\lambda \in \mathbb{X}_{\bullet}(T)$, let $\mathcal{G} r_{\lambda}=G(\mathcal{O}) t^{\lambda} G(\mathcal{O}) / G(\mathcal{O})$ be the $G(\mathcal{O})$-orbit through $t^{\lambda}$. Each $G(\mathcal{O})$-orbit of $\mathcal{G} r_{G}$ contains a unique point $t^{\lambda}$ for some dominant coweight $\lambda$. For $\lambda$ dominant, we denote the closure of $\mathcal{G} r_{\lambda}$ by $\mathcal{G} r_{\leq \lambda}$. Then each $\mathcal{G} r_{\leq \lambda}$ is a projective variety and $\mathcal{G} r_{G}$ is their union.

2.1. Weight functors and MV-filtration. In [MV07, Mirković and Vilonen introduce the weight functors

$$
H_{c}^{*}\left(S_{\mu},-\right): \mathcal{P} \rightarrow \operatorname{Mod}(\mathbb{Z}),
$$

where $S_{\mu}$ is the $U(F)$-orbit through $t^{\mu}, \mu \in \mathbb{X}_{\bullet}(T)$ (notice that in MV07, $U$ is denoted by $N$ ). They show in [MV07, Theorem 3.6] that there is a natural isomorphism of tensor functors:

$$
\left.H^{*}(-) \cong \bigoplus_{\mu \in \mathbb{X} \bullet} H_{c}^{*}(T) \text { (S },-\right): \mathcal{P} \rightarrow \operatorname{Mod}(\mathbb{Z})
$$


Moreover, under the geometric Satake isomorphism (1.1), the weight functors correspond to the weight spaces of a maximal torus $T^{\vee} \subset G^{\vee}$.

For each $\mathcal{F} \in \mathcal{P}$, consider the filtration $\left\{\mathrm{Fil}_{\geq \mu} H^{*}(\mathcal{F})\right\}$ indexed by the partially ordered set $\mathbb{X}_{\bullet}(T)$ :

$$
\operatorname{Fil}_{\geq \mu} H^{*}(\mathcal{F})=\operatorname{ker}\left(H^{*}(\mathcal{F}) \rightarrow H^{*}\left(S_{<\mu}, \mathcal{F}\right)\right),
$$

where $S_{<\mu}=\overline{S_{\mu}}-S_{\mu}$. We call this filtration the $M V$-filtration. It is functorial for $\mathcal{F} \in \mathcal{P}$. Clearly, the weight functors are the associated graded pieces of the MV-filtration. Under the geometric Satake isomorphism (1.1), there is a unique Borel subgroup $B^{\vee} \subset G^{\vee}$ containing $T^{\vee}$ such that the natural $\mathbb{X} \bullet(T)$-filtration on the $B^{\vee}$-module $H^{*}(\mathcal{F})$ coincides with the MV-filtration.

Now consider the equivariant cohomology functor:

$$
H_{T}^{*}(-): \mathcal{P} \rightarrow \operatorname{Mod}^{g r}\left(R_{T}\right) .
$$

Here $R_{T}=H_{T}^{*}(\mathrm{pt}, \mathbb{Z}) \stackrel{\sim}{\rightarrow} \operatorname{Sym}\left(\mathbb{X}^{\bullet}(T)\right)$, and $\operatorname{Mod}^{g r}\left(R_{T}\right)$ is the category of graded $R_{T}$-modules.

\subsection{Lemma. There is a natural isomorphism of functors}

$$
H_{T}^{*}(\mathcal{G} r,-) \stackrel{\sim}{\rightarrow} H^{*}(\mathcal{G} r,-) \otimes R_{T}: \mathcal{P} \rightarrow \operatorname{Mod}^{g r}\left(R_{T}\right) .
$$

Proof. The construction of weight functors (2.1) extends to the $T$-equivariant setting. For each $\mu \in \mathbb{X}_{\bullet}(T)$, the cohomology $H_{c}^{*}\left(S_{\mu}, \mathcal{F}\right)$ is concentrated in degree $\left\langle 2 \rho^{\vee}, \mu\right\rangle\left(\right.$ MV07, Theorem 3.5]), hence the equivariant cohomology $H_{T, c}^{*}\left(S_{\mu}, \mathcal{F}\right)$ is free over $R_{T}$ with a canonical isomorphism

$$
H_{T, c}^{*}\left(S_{\mu}, \mathcal{F}\right)=H_{c}^{*}\left(S_{\mu}, \mathcal{F}\right) \otimes R_{T} .
$$

There is a spectral sequence calculating $H_{T}^{*}(\mathcal{F})$ with $E_{1}$-terms $H_{T, c}^{*}\left(S_{\mu}, \mathcal{F}\right)$. Over each component of $\mathcal{G} r_{G}$, the nonzero terms of this $E_{1}$ page are all in degrees of the same parity, hence the spectral sequence degenerates. In other words, we get a $T$-equivariant version of the MV-filtration $\left\{\mathrm{Fil}_{\geq \mu} H_{T}^{*}(\mathcal{F})\right\}$ with associated graded pieces $H_{T, c}^{*}\left(S_{\mu}, \mathcal{F}\right)$.

Again, by [MV07, Theorem 3.5], $\operatorname{Fil}_{>\mu} H_{T}^{*}(\mathcal{F})$ is in degrees $>\left\langle 2 \rho^{\vee}, \mu\right\rangle$, hence we have a canonical splitting of the exact sequence

$$
0 \rightarrow \mathrm{Fil}_{>\mu} \rightarrow \mathrm{Fil}_{\geq \mu} \rightarrow H_{T, c}^{*}\left(S_{\mu}, \mathcal{F}\right) \rightarrow 0
$$

given by

$$
H_{T, c}^{*}\left(S_{\mu}, \mathcal{F}\right)=H_{c}^{\left\langle 2 \rho^{\vee}, \mu\right\rangle}\left(S_{\mu}, \mathcal{F}\right) \otimes R_{T} \rightarrow\left(H^{\left\langle 2 \rho^{\vee}, \mu\right\rangle} \mathrm{Fil}_{\geq \mu}\right) \otimes R_{T} \rightarrow \mathrm{Fil}_{\geq \mu},
$$

where $H^{k} \mathrm{Fil}_{\geq \mu}$ means the degree $k$ part of $\mathrm{Fil}_{\geq \mu}$. Therefore, the $T$-equivariant MV-filtration on $H_{T}^{*}(\mathcal{F})$ also has a canonical splitting:

$$
H_{T}^{*}(-) \cong \bigoplus_{\mu \in \mathbb{X} \bullet(T)} H_{T, c}^{*}\left(S_{\mu},-\right): \mathcal{P} \rightarrow \operatorname{Mod}^{g r}\left(R_{T}\right) .
$$

Combining (2.4), (2.5) and (2.2), we get isomorphisms of functors $\mathcal{P} \rightarrow$ $\operatorname{Mod}^{g r}\left(R_{T}\right)$ :

$$
H_{T}^{*}(-) \cong \bigoplus_{\mu \in \mathbb{X}_{\bullet}(T)} H_{T, c}^{*}\left(S_{\mu},-\right) \cong \bigoplus_{\mu \in \mathbb{X}_{\bullet}(T)} H_{c}^{*}\left(S_{\mu},-\right) \otimes R_{T} \cong H^{*}(-) \otimes R_{T} .
$$


2.3. Monoidal structure on $H_{T}^{*}(-)$. Although the isomorphism in Lemma 2.2 equips $H_{T}^{*}(-)$ with a monoidal structure, this monoidal structure is, a priori, not what we want to use later. We recall here another monoidal structure on $H_{T}^{*}(-)$, following [MV07, $\S 5, \S 6]$. First recall the definition of the convolution product $*$ on $\mathcal{P}$, following [MV07, §4]. Consider the diagram

$$
\mathcal{G} r_{G} \times \mathcal{G} r_{G} \stackrel{p}{\leftarrow} G(F) \times \mathcal{G} r_{G} \stackrel{q}{\rightarrow} G(F) \stackrel{G(\mathcal{O})}{\times} \mathcal{G} r_{G} \stackrel{m_{G}}{\longrightarrow} \mathcal{G} r_{G} .
$$

Here $p$ is the natural projection morphism, $q$ is the quotient map by the right $G(\mathcal{O})$ action on $G(F) \times \mathcal{G} r_{G}$ given by $(x, y) \cdot g=\left(x g, g^{-1} y\right)$ and $m_{G}$ is induced by the multiplication on $G(F)$. By definition,

$$
\mathcal{F}_{1} * \mathcal{F}_{2}=m_{G, *} \widetilde{\mathcal{F}}
$$

where $\widetilde{\mathcal{F}}$ is the unique perverse sheaf on $G(F) \stackrel{G(\mathcal{O})}{\times} \mathcal{G} r_{G}$ such that

$$
q^{*}(\widetilde{\mathcal{F}})=p^{* p} H^{0}\left(\mathcal{F}_{1} \stackrel{\mathbb{L}}{\otimes} \mathcal{F}_{2}\right) .
$$

Hence

$$
H_{T}^{*}\left(\mathcal{F}_{1} * \mathcal{F}_{2}\right) \cong H_{T}^{*}\left(G(F) \stackrel{G(\mathcal{O})}{\times} \mathcal{G} r_{G}, \widetilde{\mathcal{F}}\right) .
$$

Let $X=\mathbb{A}_{\mathbb{C}}^{1}$. Recall that the global counterpart $\mathcal{G} r_{X}$ of $\mathcal{G} r_{G}$ classifies triples $(x, \mathcal{E}, \tau)$ where $x \in X, \mathcal{E}$ is a $G$-torsor over $X$ and $\tau$ is a trivialization of $\mathcal{E}$ over $X-\{x\}$. The global counterpart of $G(F) \stackrel{G(\mathcal{O})}{\times} \mathcal{G} r_{G}$ is the space $\mathcal{G} r_{X} \widetilde{\times} \mathcal{G} r_{X}$ which classifies tuples $\left(x_{1}, x_{2}, \mathcal{E}_{1}, \mathcal{E}_{2}, \tau_{1}, \tau_{2}\right)$ where $x_{1}, x_{2} \in X, \mathcal{E}_{1}, \mathcal{E}_{2}$ are $G$-torsors over $X, \tau_{1}$ is a trivialization of $\mathcal{E}_{1}$ on $X-\left\{x_{1}\right\}$ and $\tau_{2}$ is an isomorphism of $G$-torsors $\left.\left.\mathcal{E}_{1}\right|_{X-\left\{x_{2}\right\}} \stackrel{\sim}{\rightarrow} \mathcal{E}_{2}\right|_{X-\left\{x_{2}\right\}}$. For $x_{1} \neq x_{2}$, the fiber $\left(\mathcal{G} r_{X} \widetilde{\times} \mathcal{G} r_{X}\right)_{x_{1}, x_{2}}$ is isomorphic to $\mathcal{G} r_{G} \times \mathcal{G} r_{G}$; while for $x_{1}=x_{2}$, the fiber $\left(\mathcal{G} r_{X} \widetilde{\times} \mathcal{G} r_{X}\right)_{x_{1}, x_{2}}$ is isomorphic to $G(F) \stackrel{G(\mathcal{O})}{\times}$ $\mathcal{G} r_{G}$.

Given objects $\mathcal{F}_{1}, \mathcal{F}_{2} \in \mathcal{P}$, we can "spread them over the curve $X$ " to get perverse sheaves $\mathcal{K}_{1}, \mathcal{K}_{2}$ on $\mathcal{G} r_{X}$; we can also mimic the construction of $\widetilde{\mathcal{F}}$ to get a perverse sheaf $\widetilde{\mathcal{K}}$ on $\mathcal{G} r_{X} \widetilde{\times} \mathcal{G} r_{X}$ (see [MV07, §5] for details). The restriction of $\widetilde{\mathcal{K}}$ on $\left(\mathcal{G} r_{X} \tilde{\times} \mathcal{G} r_{X}\right)_{x_{1}, x_{2}}$ can be identified with ${ }^{p} H^{0}\left(\mathcal{F}_{1} \stackrel{\mathbb{Z}}{\otimes} \mathcal{F}_{2}\right)$ when $x_{1} \neq x_{2}$ and with $\widetilde{\mathcal{F}}$ when $x_{1}=x_{2}$, under the above identification of fibers. In MV07, Lemma 6.1, eqn.(6.4)], it is shown that the direct image sheaves $\mathbf{R}^{i} \widetilde{\pi}_{*}(\widetilde{\mathcal{K}})$ are constant (as $T$ equivariant sheaves, as we can easily see), where $\tilde{\pi}: \mathcal{G} r_{X} \widetilde{\times} \mathcal{G} r_{X} \rightarrow X^{2}$ is the natural projection. In other words, we have a co-specialization isomorphism from the stalks of $\mathbf{R}^{i} \widetilde{\pi}_{*} \widetilde{\mathcal{K}}$ along the diagonal $\Delta(X) \subset X^{2}$ to its stalks over $X^{2}-\Delta(X)$. Using the above identifications, the co-specialization map takes the form:

$$
\mathrm{Sp}_{\widetilde{\mathcal{K}}}^{*}: H_{T}^{*}\left(G(F) \stackrel{G(\mathcal{O})}{\times} \mathcal{G} r_{G}, \widetilde{\mathcal{F}}\right) \stackrel{\sim}{\rightarrow} H_{T}^{*}\left(\mathcal{G} r_{G} \times \mathcal{G} r_{G},{ }^{p} H^{0}\left(\mathcal{F}_{1} \stackrel{\mathbb{L}}{\otimes} \mathcal{F}_{2}\right)\right) .
$$

The argument of [MV07, Lemma 6.1, eqn.(6.3)] has an obvious $T$-equivariant version (using Lemma 2.2), hence

$$
H_{T}^{*}\left(\mathcal{G} r_{G} \times \mathcal{G} r_{G},{ }^{p} H^{0}\left(\mathcal{F}_{1} \stackrel{\mathbb{Q}}{\otimes} \mathcal{F}_{2}\right)\right) \cong H_{T}^{*}\left(\mathcal{F}_{1}\right) \otimes_{R_{T}} H_{T}^{*}\left(\mathcal{F}_{2}\right) .
$$

Combining (2.7), (2.6) and (2.8), we get the monoidal structure

$$
H_{T}^{*}\left(\mathcal{F}_{1} * \mathcal{F}_{2}\right) \stackrel{\sim}{\rightarrow} H_{T}^{*}\left(\mathcal{F}_{1}\right) \otimes_{R_{T}} H_{T}^{*}\left(\mathcal{F}_{2}\right) .
$$


2.4. Lemma. Under the monoidal structure of $H_{T}^{*}(-)$ introduced in $\$ 2.3$ and the monoidal structure of $H^{*}(-) \otimes R_{T}$ induced from that of $H^{*}(-)$, the natural isomorphism in Lemma 2.2 is a monoidal isomorphism. In particular, the monoidal structure on $H_{T}^{*}(-)$ introduced in $\$ 2.3$ is also a tensor structure (i.e., it is compatible with the commutativity constraint 2).

Proof. Using the same argument of [MV07, §6], one can show that the sum of the $T$-equivariant weight functors has a natural monoidal structure. Moreover, the isomorphism in (2.5) is a monoidal isomorphism. Using (2.4) and the argument of [MV07, Proposition 6.4], it is easy to see that

$$
\bigoplus_{\mu \in \mathbb{\mathbb { X } _ { \bullet }}(T)} H_{T, c}^{*}\left(S_{\mu},-\right) \cong \bigoplus_{\mu \in \mathbb{X}_{\bullet}(T)} H_{c}^{*}\left(S_{\mu},-\right) \otimes R_{T}: \mathcal{P} \rightarrow \operatorname{Mod}^{\mathbb{X} \bullet}(T)\left(R_{T}\right)
$$

as monoidal functors. The natural isomorphism $H_{T}^{*}(-) \cong H^{*}(-) \otimes R_{T}$ in Lemma 2.2 is given by the composition of monoidal isomorphisms (2.5), (2.9) and (2.2), hence it is also a monoidal isomorphism.

2.5. Remark. Similarly, we can consider

$$
H_{G(\mathcal{O})}^{*}(-)=H_{G}^{*}(-): \mathcal{P} \rightarrow \operatorname{Mod}^{g r}\left(R_{G}\right) .
$$

Here $R_{G}=H_{G}^{*}(\mathrm{pt}, \mathbb{Z})$. Using the argument of [MV07, Proposition 6.1], one can show that $H_{G}^{*}(-)$ is also a tensor functor. Further argument shows that it is actually a fiber functor (i.e., it is exact and faithful). By [Sa72, this fiber functor defines a $G^{\vee}$-torsor $\mathcal{E}$ over $\operatorname{Spec} R_{G}$ (here we use the fact that $G^{\vee}$ is flat over $\mathbb{Z}$ ). Moreover, by Lemma 2.2. the pull back of $\mathcal{E}$ to $\operatorname{Spec} R_{T}$ admits a canonical trivialization. However, $\mathcal{E}$ itself does not have a canonical trivialization.

2.6. Equivariant (co)homology of $\mathcal{G} r_{G}$. Since $H_{T}^{*}\left(\mathcal{G} r_{G}\right)=H_{T \cap K}^{*}(\Omega K)$ and $\Omega K$ is a homotopy commutative $H$-space, $H_{T}^{*}\left(\mathcal{G} r_{G}\right)$ is naturally a commutative and cocommutative Hopf algebra over $R_{T}$. By the cell-decomposition of $\mathcal{G} r_{G}, H_{T}^{*}\left(\mathcal{G} r_{G}\right)$ is a free $R_{T}$-module concentrated in even degrees. We denote the coproduct on $H_{T}^{*}\left(\mathcal{G} r_{G}\right)$ by $\Delta$.

We define the $T$-equivariant homology

$$
H_{*}^{T}\left(\mathcal{G} r_{G}\right):=\operatorname{Hom}_{R_{T}}\left(H_{T}^{*}\left(\mathcal{G} r_{G}\right), R_{T}\right)^{g r}
$$

to be the graded dual of $H_{T}^{*}\left(\mathcal{G} r_{G}\right)$ as an $R_{T}$-module, i.e., $H_{n}^{T}\left(\mathcal{G} r_{G}\right)$ sends $H_{T}^{i}\left(\mathcal{G} r_{G}\right)$ to $R_{T}^{i-n}$. Note that $H_{*}^{T}\left(\mathcal{G} r_{G}\right) \cong \lim H_{*}^{T}\left(\mathcal{G} r_{\leq \lambda}\right)$ where $H_{*}^{T}\left(\mathcal{G} r_{\leq \lambda}\right)=H_{T}^{-*}\left(\mathcal{G} r_{\leq \lambda}, \mathbb{D}_{\leq \lambda}\right)$ $\left(\mathbb{D}_{\leq \lambda}\right.$ is the dualizing complex of $\mathcal{G} r_{\leq \lambda}$ with the canonical $T$-equivariant structure).

As the $R_{T}$-dual of $H_{T}^{*}\left(\mathcal{G} r_{G}\right)$, the $T$-equivariant homology $H_{*}^{T}\left(\mathcal{G} r_{G}\right)$ is also a commutative and co-commutative Hopf algebra over $R_{T}$. The multiplication on $H_{*}^{T}\left(\mathcal{G} r_{G}\right)$ is identified with the Pontryagin product on $H_{*}^{T \cap K}(\Omega K)$, and is denoted by $\wedge$.

Similarly, we can define the $G$-equivariant homology $H_{*}^{G}\left(\mathcal{G} r_{G}\right)$ of $\mathcal{G} r_{G}$ either as the $R_{G}$-dual of $H_{G}^{*}\left(\mathcal{G} r_{G}\right)$ or the direct limit of $H_{G}^{-*}\left(\mathcal{G} r_{\leq \lambda}, \mathbb{D}_{\leq \lambda}\right)$. This is a Hopf algebra over $R_{G}$.

Next, we consider the action of $H_{T}^{*}\left(\mathcal{G} r_{G}\right)$ on the fiber functor $H_{T}^{*}\left(\mathcal{G} r_{G},-\right)$ via cup product.

\footnotetext{
${ }^{2}$ See [MV07 Section 6] for the definition of the commutativity constraints on $\mathcal{P}$.
} 
2.7. Proposition. For any $\mathcal{F}_{1}, \mathcal{F}_{2} \in \mathcal{P}$, and any $h \in H_{T}^{*}\left(\mathcal{G} r_{G}\right)$, we have a commutative diagram

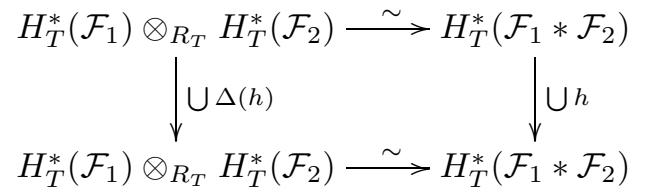

where the horizontal maps are given by the tensor structure of the functor $H_{T}^{*}(-)$ (see the proof of Lemma 2.2).

A similar statement also holds for $G$-equivariant cohomology $H_{G}^{*}$.

Proof. From (2.6) we see that the action of $h \in H_{T}^{*}\left(\mathcal{G r}_{G}\right)$ on $H_{T}^{*}\left(\mathcal{F}_{1} * \mathcal{F}_{2}\right)$ is the same as the action of $m_{G}^{*}(h) \in H_{T}^{*}\left(G(F) \stackrel{G(\mathcal{O})}{\times} \mathcal{G} r_{G}\right)$ on $H_{T}^{*}\left(G(F) \stackrel{G(\mathcal{O})}{\times} \mathcal{G} r_{G}, \widetilde{\mathcal{F}}\right)$.

Let us keep track of the cup product by the cohomology of the relevant spaces in the construction of the tensor structure in $\$ 2.3$. Since the morphism $\tilde{\pi}: \mathcal{G} r_{X} \widetilde{\times} \mathcal{G} r_{X} \rightarrow$ $X^{2}$ is stratified by affine space bundles (see MV07, Lemma 6.1, argument for (6.4)]), an easy spectral sequence argument shows that the sheaves $\mathbf{R}^{i} \widetilde{\pi}_{*} \mathbb{Z}$ are locally constant, hence constant. In other words, we also have a co-specialization isomorphism from the stalks of $\mathbf{R}^{i} \widetilde{\pi}_{*} \mathbb{Z}$ along $\Delta(X)$ to stalks elsewhere, i.e.,

$$
\mathrm{Sp}^{*}: H_{T}^{*}\left(G(F) \stackrel{G(\mathcal{O})}{\times} \mathcal{G} r_{G}\right) \stackrel{\sim}{\longrightarrow} H_{T}^{*}\left(\mathcal{G} r_{G} \times \mathcal{G} r_{G}\right) .
$$

Moreover, by the naturality of co-specialization maps, (2.7) and (2.11) are compatible under the cup product:

$$
\operatorname{Sp}_{\widetilde{\mathcal{K}}}^{*}(\widetilde{h} \cup v)=\operatorname{Sp}^{*}(\widetilde{h}) \cup \operatorname{Sp}_{\widetilde{\mathcal{K}}}^{*}(v),
$$

for any $\widetilde{h} \in H_{T}^{*}\left(G(F) \stackrel{G(\mathcal{O})}{\times} \mathcal{G} r_{G}\right), v \in H_{T}^{*}\left(G(F) \stackrel{G(\mathcal{O})}{\times} \mathcal{G} r_{G}, \widetilde{\mathcal{F}}\right)$.

From the above discussion, the cup product action of $h \in H_{T}^{*}\left(\mathcal{G} r_{G}\right)$ on $H_{T}^{*}\left(\mathcal{F}_{1}\right.$ * $\left.\mathcal{F}_{2}\right)$, when transported to $H_{T}^{*}\left(\mathcal{F}_{1}\right) \otimes_{R_{T}} H_{T}^{*}\left(\mathcal{F}_{2}\right)$ under the isomorphism given by the tensor structure of $H_{T}^{*}(-)$, is the cup product action of the class $\operatorname{Sp}^{*}\left(m_{G}^{*}(h)\right) \in$ $H_{T}^{*}\left(\mathcal{G} r_{G} \times \mathcal{G} r_{G}\right)=H_{T}^{*}\left(\mathcal{G} r_{G}\right) \otimes_{R_{T}} H_{T}^{*}\left(\mathcal{G} r_{G}\right)$. Therefore, to prove the proposition, it suffices to show that

$$
\Delta=\mathrm{Sp}^{*} m_{G}^{*}: H_{T}^{*}\left(\mathcal{G} r_{G}\right) \rightarrow H_{T}^{*}\left(\mathcal{G} r_{G} \times \mathcal{G} r_{G}\right) .
$$

Recall that the coproduct $\Delta$ is induced from the multiplication $m_{K}: \Omega K \times \Omega K \rightarrow$ $\Omega K$, hence

$$
(\iota \times \iota)^{*} \Delta=m_{K}^{*} \iota^{*}: H_{T}^{*}\left(\mathcal{G} r_{G}\right) \rightarrow H_{T \cap K}^{*}(\Omega K \times \Omega K) .
$$

Now we construct a map $\widetilde{\iota}: \Omega K \times \Omega K \times X^{2} \rightarrow \mathcal{G} r_{X} \widetilde{\times} \mathcal{G} r_{X}$. For a point $\left(\gamma_{1}, \gamma_{2}\right.$ : $\left.S^{1} \rightarrow K, x_{1}, x_{2}\right) \in \Omega K \times \Omega K \times X^{2}$, we consider the complexifications $\gamma_{1}^{\mathbb{C}}, \gamma_{2}^{\mathbb{C}}:$ $\mathbb{A}^{1}-\{0\} \rightarrow G$ (recall that $\gamma_{i}$ are polynomial maps so that the complexifications make sense). Let $\gamma_{i, x_{i}}^{\mathbb{C}}: \mathbb{A}^{1}-\left\{x_{i}\right\} \rightarrow G$ be the composition of the translation $\mathbb{A}^{1}-\left\{x_{i}\right\} \stackrel{\sim}{\rightarrow} \mathbb{A}^{1}-\{0\}$ with $\gamma_{i}^{\mathbb{C}}$. We define

$$
\widetilde{\iota}\left(\gamma_{1}, \gamma_{2}, x_{1}, x_{2}\right)=\left(x_{1}, x_{2}, \mathcal{E}_{1}=\mathcal{E}^{\text {triv }}, \mathcal{E}_{2}=\mathcal{E}^{\text {triv }}, \tau_{1}, \tau_{2}\right)
$$

where $\mathcal{E}^{\text {triv }}$ is the trivial $G$-torsor on $X$, and the isomorphism $\tau_{i}$ (viewed as an automorphism of $\mathcal{E}^{\text {triv }}$ over $\left.X-\left\{x_{i}\right\}\right)$ is given by $\gamma_{i, x_{i}}^{\mathbb{C}}: X-\left\{x_{i}\right\} \rightarrow G$ for $i=1,2$.

The restriction of $\widetilde{\iota}$ to points $x_{1}=x_{2}$ can be identified with the map $\iota_{(2)}$ : $\Omega K \times \Omega K \stackrel{\iota \times \iota}{\longrightarrow} G(F) \times \mathcal{G} r_{G} \stackrel{q}{\rightarrow} G(F) \stackrel{G(\mathcal{O})}{\times} \mathcal{G} r_{G}$; the restriction of $\widetilde{\iota}$ to $x_{1} \neq x_{2}$ is 
exactly the map $\iota \times \iota: \Omega K \times \Omega K \rightarrow \mathcal{G} r_{G} \times \mathcal{G} r_{G}$. We view $\Omega K \times \Omega K \times X^{2}$ as a constant family over $X^{2}$, then $\widetilde{\iota}$ commutes with the co-specialization maps, i.e., we have a commutative diagram

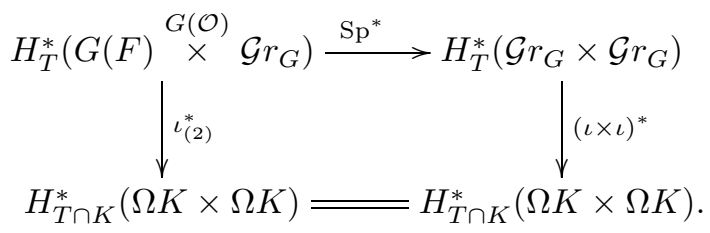

On the other hand, we also have a commutative diagram

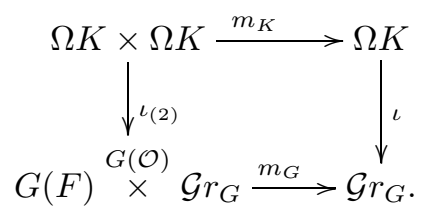

Combining the two commutative diagrams, we get

$$
(\iota \times \iota)^{*} \mathrm{Sp}^{*} m_{G}^{*}=\iota_{(2)}^{*} m_{G}^{*}=m_{K}^{*} \iota^{*} .
$$

In view of (2.13), we get

$$
(\iota \times \iota)^{*} \Delta=(\iota \times \iota)^{*} \mathrm{Sp}^{*} m_{G}^{*} .
$$

Since $(\iota \times \iota)^{*}$ is an isomorphism, the equality (2.12) follows. This finishes the proof of the proposition.

\section{Relating $H_{*}^{T}\left(\mathcal{G} r_{G}\right)$ TO The Langlands DuAl GRouP}

Consider the fiber functor

$$
H_{T}^{*}(-) \otimes_{R_{T}} H_{*}^{T}\left(\mathcal{G} r_{G}\right): \mathcal{P} \rightarrow \operatorname{Mod}^{g r}\left(H_{*}^{T}\left(\mathcal{G} r_{G}\right)\right)
$$

We now construct a canonical tensor automorphism $\sigma_{\text {can }}$ of this functor. For any $\mathcal{F} \in \mathcal{P}$, the action of $\sigma_{\text {can }}$ on $H_{T}^{*}(\mathcal{F}) \otimes_{R_{T}} H_{*}^{T}\left(\mathcal{G} r_{G}\right)$ is given by

$$
\sigma_{c a n}(v \otimes h)=\sum_{i}\left(h^{i} \cup v\right) \otimes\left(h_{i} \wedge h\right),
$$

where $v \in H_{T}^{*}(\mathcal{F}), h \in H_{*}^{T}\left(\mathcal{G} r_{G}\right)$ and $\left\{h^{i}\right\},\left\{h_{i}\right\}$ are dual bases of the free $R_{T^{-}}$ modules $H_{T}^{*}\left(\mathcal{G} r_{G}\right)$ and $H_{*}^{T}\left(\mathcal{G} r_{G}\right)$. One readily checks that $\sigma_{c a n}$ does not depend on the choice of the dual bases $\left\{h^{i}\right\},\left\{h_{i}\right\}$.

3.1. Lemma. The natural transformation $\sigma_{\text {can }}$ is a tensor automorphism of the fiber functor $H_{T}^{*}(-) \otimes_{R_{T}} H_{*}^{T}\left(\mathcal{G} r_{G}\right)$.

Proof. Let $\mathcal{F}_{1}, \mathcal{F}_{2} \in \mathcal{P}$ and $v_{i} \in H_{T}^{*}\left(\mathcal{F}_{i}\right)$ for $i=1,2$. We have to show that

$$
\sigma_{c a n}\left(v_{1} \otimes v_{2}\right)=\sigma_{c a n}\left(v_{1}\right) \otimes \sigma_{c a n}\left(v_{2}\right) .
$$

Here we view $v_{1} \otimes v_{2}$ as an element in $H_{T}^{*}\left(\mathcal{F}_{1} * \mathcal{F}_{2}\right)$ under the identification $H_{T}^{*}\left(\mathcal{F}_{1}\right) \otimes_{R_{T}} H_{T}^{*}\left(\mathcal{F}_{2}\right) \cong H_{T}^{*}\left(\mathcal{F}_{1} * \mathcal{F}_{2}\right)$. 
On one hand, we have

$$
\begin{aligned}
\sigma_{c a n}\left(v_{1}\right) \otimes \sigma_{c a n}\left(v_{2}\right) & =\sum_{i, j}\left(h^{i} \cup v_{1} \otimes h_{i}\right) \otimes\left(h^{j} \cup v_{2}\right) \otimes h_{j} \\
& =\sum_{i, j}\left(\left(h^{i} \cup v_{1}\right) \otimes\left(h^{j} \cup v_{2}\right)\right) \otimes\left(h_{i} \wedge h_{j}\right) .
\end{aligned}
$$

On the other hand, by Proposition 2.7 .

$$
\sigma_{c a n}\left(v_{1} \otimes v_{2}\right)=\sum_{k}\left(\Delta\left(h^{k}\right)\left(v_{1} \otimes v_{2}\right)\right) \otimes h_{k} .
$$

Since the coproduct $\Delta$ on $H_{T}^{*}\left(\mathcal{G} r_{G}\right)$ is adjoint to the Pontryagin product $\wedge$ on $H_{*}^{T}\left(\mathcal{G} r_{G}\right)$, the two expressions (3.1) and (3.2) are the same.

By this lemma and the Tannakian formalism, $\sigma_{\text {can }}$ defines an $H_{*}^{T}\left(\mathcal{G} r_{G}\right)$-valued point of the group scheme $\operatorname{Aut}^{\otimes}\left(H_{T}^{*}\right)$, which is canonically isomorphic to $G^{\vee} \times$ Spec $R_{T}$ by Lemma 2.2.

$$
\widetilde{\sigma}^{T}: \operatorname{Spec} H_{*}^{T}\left(\mathcal{G} r_{G}\right) \rightarrow G^{\vee} \times \operatorname{Spec} R_{T} .
$$

We also have the non-equivariant counterpart:

$$
\widetilde{\sigma}: \operatorname{Spec} H_{*}\left(\mathcal{G} r_{G}\right) \rightarrow G^{\vee} .
$$

3.2. Lemma. The morphisms $\widetilde{\sigma}^{T}$ and $\widetilde{\sigma}$ as in (3.3) and (3.4) are homomorphisms of group schemes over $R_{T}$ and $\mathbb{Z}$.

Proof. We give the argument for the non-equivariant version, and the equivariant version is similar. We abbreviate $H_{*}\left(\mathcal{G} r_{G}\right)$ by $H_{*}$ and $H^{*}\left(\mathcal{G} r_{G}\right)$ by $H^{*}$. We need to check that the following diagram is commutative

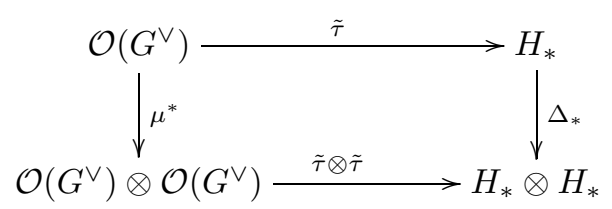

where $\mu^{*}$ and $\Delta_{*}$ are the coproducts on $\mathcal{O}\left(G^{\vee}\right)$ and $H_{*}$. The composition $(\tilde{\tau} \otimes \tilde{\tau}) \circ \mu^{*}$ corresponds to the following automorphism of the tensor functor $H^{*}(-) \otimes H_{*} \otimes H_{*}$ :

$$
\begin{aligned}
H^{*}(\mathcal{F}) \otimes H_{*} \otimes H_{*} & \rightarrow H^{*}(\mathcal{F}) \otimes H_{*} \otimes H_{*}, \\
v \otimes 1 \otimes 1 & \mapsto \sum_{i, j}\left(h^{i} \cup h^{j} \cup v\right) \otimes h_{i} \otimes h_{j},
\end{aligned}
$$

for $v \in H^{*}(\mathcal{F})$ and $\left\{h^{i}\right\},\left\{h_{i}\right\}$ dual bases of $H^{*}$ and $H_{*}$ as before. On the other hand, the composition $\Delta_{*} \circ \tilde{\tau}$ is given by the automorphism

$$
\begin{aligned}
H^{*}(\mathcal{F}) \otimes H_{*} \otimes H_{*} & \rightarrow H^{*}(\mathcal{F}) \otimes H_{*} \otimes H_{*}, \\
v \otimes 1 \otimes 1 & \mapsto \sum_{i}\left(h^{k} \cup v\right) \otimes \Delta_{*}\left(h_{k}\right) .
\end{aligned}
$$

Since $\Delta_{*}: H_{*} \rightarrow H_{*} \otimes H_{*}$ is adjoint to $\cup: H^{*} \otimes H^{*} \rightarrow H^{*}$, the two expressions (3.5) and (3.6) are the same.

\subsection{Proposition.}

(1) The homomorphism $\widetilde{\sigma}^{T}$ factors through $B^{\vee} \times \operatorname{Spec} R_{T}$; 
(2) We have a natural isomorphism of group schemes $\operatorname{Spec} H_{0}\left(\mathcal{G} r_{G}\right) \stackrel{\sim}{\rightarrow} Z^{\vee}$, the center of $G^{\vee}$;

(3) If $G$ is simply-connected, $\widetilde{\sigma}$ factors through $U^{\vee}$;

(4) In general, let $G^{s c}$ be the simply-connected cover of $G^{\text {der }}$, and we identify the neutral component $\mathcal{G} r_{G}^{0}$ with $\mathcal{G} r_{G s}$. Then we have a natural isomorphism of Hopf algebras:

$$
H_{*}\left(\mathcal{G} r_{G}\right) \cong H_{0}\left(\mathcal{G} r_{G}\right) \otimes H_{*}\left(\mathcal{G} r_{G^{s c}}\right) .
$$

The morphism $\widetilde{\sigma}:$ Spec $H_{*}\left(\mathcal{G} r_{G}\right) \rightarrow B^{\vee}$ factors as

$\operatorname{Spec} H_{*}\left(\mathcal{G} r_{G}\right) \rightarrow \operatorname{Spec} H_{0}\left(\mathcal{G} r_{G}\right) \times \operatorname{Spec} H_{*}\left(\mathcal{G} r_{G^{s c}}\right) \stackrel{*}{\rightarrow} Z^{\vee} \times U^{\vee} \hookrightarrow B^{\vee}$,

where the starred arrow is the product of the morphisms in (2) and (3). Here we have identified unipotent radicals $U^{\vee}$ of $B^{\vee}$ for $G^{\vee}$ and $\left(G^{s c}\right)^{\vee}=$ $\left(G^{\vee}\right)^{a d}$.

Proof. (1) The action of $\sigma_{c a n}$ preserves the MV-filtration on $H_{T}^{*}(-) \otimes_{R_{T}} H_{*}^{T}\left(\mathcal{G} r_{G}\right)$, which implies that $\widetilde{\sigma}^{T}$ factors through $B^{\vee} \times \operatorname{Spec} R_{T}$.

(2) The component group $\pi_{0}\left(\mathcal{G} r_{G}\right) \cong \pi_{1}(K) \cong \mathbb{X} \cdot(T) / \mathbb{Z} \Phi$ (here $\mathbb{Z} \Phi$ is the coroot lattice of $G)$. Therefore, $H_{0}\left(\mathcal{G} r_{G}\right)$ is the group algebra of $\mathbb{X} \bullet(T) / \mathbb{Z} \Phi$, hence isomorphic to $\mathcal{O}\left(Z^{\vee}\right)$ as Hopf algebras.

(3) and (4) Since the tensor automorphism $\sigma_{c a n}$ preserves the MV-filtration, it induces a tensor automorphism $\bar{\sigma}_{c a n}$ on the associated graded pieces, i.e., on the tensor functor

$$
\bigoplus_{\mu \in \mathbb{X} \bullet} H_{c}^{*}\left(S_{\mu},-\right) \otimes H_{*}\left(\mathcal{G} r_{G}\right): \mathcal{P} \rightarrow \operatorname{Mod}^{\mathbb{X} \bullet}(T)\left(H_{*}\left(\mathcal{G} r_{G}\right)\right) .
$$

The tensor automorphism $\bar{\sigma}_{\text {can }}$ gives a homomorphism Spec $H_{*}\left(\mathcal{G} r_{G}\right) \rightarrow T^{\vee}$ which is clearly the same as the composition Spec $H_{*}\left(\mathcal{G} r_{G}\right) \stackrel{\widetilde{\sigma}}{\rightarrow} B^{\vee} \stackrel{\pi}{\rightarrow} T^{\vee}$. On the other hand, since $H_{c}^{*}\left(S_{\mu}, \mathcal{F}\right)$ is concentrated in one degree, the action of $\bar{\sigma}_{c a n}$ on it is given by

$$
v \mapsto\left(\bar{\mu}^{*} \cup v\right) \otimes \bar{\mu} \in H_{c}^{*}\left(S_{\mu}, \mathcal{F}\right) \otimes H_{0}\left(\mathcal{G} r_{G}\right) .
$$

Here $v \in H_{c}^{*}\left(S_{\mu}, \mathcal{F}\right), \bar{\mu}$ is the image of $\mu \in \mathbb{X} \bullet(T)$ in $\mathbb{X}_{\bullet}(T) / \mathbb{Z} \Phi^{\vee} \subset \mathbb{Z}\left[\mathbb{X} \bullet(T) / \mathbb{Z} \Phi^{\vee}\right]=$ $H_{0}\left(\mathcal{G} r_{G}\right)$, and $\bar{\mu}^{*}$ is the element in $H^{0}\left(\mathcal{G} r_{G}\right)$ that takes value 1 on the component corresponding to $\bar{\mu}$ and 0 on other components. This means that the tensor automorphism $\bar{\sigma}_{\text {can }}$ in fact comes from a tensor automorphism of the functor

$$
\bigoplus_{\mu \in \mathbb{X} \bullet(T)} H_{c}^{*}\left(S_{\mu},-\right) \otimes H_{0}\left(\mathcal{G} r_{G}\right): \mathcal{P} \rightarrow \operatorname{Mod}^{\mathbb{X} \bullet}(T)\left(H_{0}\left(\mathcal{G} r_{G}\right)\right)
$$

followed by the functor $\operatorname{Mod}^{\mathbb{X} \bullet(T)}\left(H_{0}\left(\mathcal{G} r_{G}\right)\right) \rightarrow \operatorname{Mod}^{\mathbb{X} \bullet(T)}\left(H_{*}\left(\mathcal{G} r_{G}\right)\right)$ given by $\otimes_{H_{0}} H_{*}\left(\mathcal{G} r_{G}\right)$. In other words, there is a commutative diagram

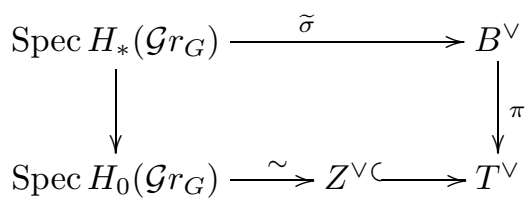

which implies that $\widetilde{\sigma}$ factors through $\pi^{-1}\left(Z^{\vee}\right)=Z^{\vee} \times U^{\vee} \subset B^{\vee}$. 
Now if $G=G^{s c}, Z^{\vee}$ is trivial and the map $H_{*}\left(\mathcal{G} r_{G}\right) \rightarrow B^{\vee}$ factors through $U^{\vee}$. This proves (3). In general, by the functoriality of the geometric Satake isomorphism for the homomorphism $G^{s c} \rightarrow G$, we have a commutative diagram

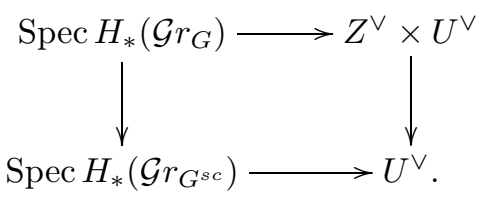

Combining (3.7) and (3.8), the assertion is proved.

3.4. Remark. The composition

$$
\operatorname{Spec} H_{*}^{T}\left(\mathcal{G} r_{G}\right) \stackrel{\tilde{\sigma}^{T}}{\longrightarrow} B^{\vee} \times \operatorname{Spec} R_{T} \rightarrow T^{\vee} \times \operatorname{Spec} R_{T}
$$

can be interpreted via equivariant localization. In fact, as we remarked in the proof of Proposition 3.3(3),(4), the above morphism Spec $H_{*}^{T}\left(\mathcal{G} r_{G}\right) \rightarrow T^{\vee} \times \operatorname{Spec} R_{T}$ comes from the tensor automorphism $\bar{\sigma}_{c a n}$ of the $T$-equivariant weight functors (2.9). On the other hand, the cup product action of $H_{T}^{*}\left(\mathcal{G} r_{G}\right)$ on $H_{T, c}^{*}\left(S_{\lambda},-\right)$ factors through the restriction map $i_{\lambda}^{*}: H_{T}^{*}\left(\mathcal{G} r_{G}\right) \rightarrow H_{T}^{*}\left(S_{\lambda}\right) \stackrel{\sim}{\longrightarrow} H_{T}^{*}\left(\left\{t^{\lambda}\right\}\right)$ (the second arrow is an isomorphism because $S_{\lambda}$ contracts to $t^{\lambda}$ under a $\mathbb{G}_{m}$-action). Therefore, for $v_{\lambda} \in H_{T, c}^{*}\left(S_{\lambda}, \mathcal{F}\right)$, we have

$$
\bar{\sigma}_{c a n}\left(v_{\lambda}\right)=\sum_{i}\left(i_{\lambda}^{*}\left(h^{i}\right) \cup v_{\lambda}\right) \otimes h_{i}=v_{\lambda} \otimes i_{\lambda, *}(1),
$$

where $i_{\lambda, *}: R_{T}=H_{*}^{T}\left(\left\{t^{\lambda}\right\}\right) \rightarrow H_{*}^{T}\left(\mathcal{G} r_{G}\right)$ is the adjoint of $i_{\lambda}^{*}$. The product of $i_{\lambda}^{*}$ is the equivariant localization map:

$$
L o c^{*}=\prod_{\lambda \in \mathbb{X} \bullet(T)} i_{\lambda}^{*}: H_{T}^{*}\left(\mathcal{G} r_{G}\right) \rightarrow \prod H_{T}^{*}\left(\left\{t^{\lambda}\right\}\right) .
$$

Let

$$
L o c_{*}=\sum_{\lambda \in \mathbb{X}_{\bullet}(T)} i_{\lambda, *}: R_{T}\left[\mathbb{X}_{\bullet}(T)\right] \rightarrow H_{*}^{T}\left(\mathcal{G} r_{G}\right)
$$

be the adjoint of $L o c^{*}$, where $R_{T}\left[\mathbb{X}_{\bullet}(T)\right]$ is the $R_{T}$-valued group ring of the abelian group $\mathbb{X}_{\bullet}(T)$. The above discussion shows that the morphism (3.9) coincides with

$$
\operatorname{Spec}\left(L o c_{*}\right): \operatorname{Spec} H_{*}^{T}\left(\mathcal{G} r_{G}\right) \rightarrow \operatorname{Spec} R_{T}\left[\mathbb{X}_{\bullet}(T)\right]=T^{\vee} \times \operatorname{Spec} R_{T}
$$

\section{REMARKS ON LINE BUNDLES}

From this section on, we assume that $G^{d e r}$ is almost simple. In this case, it is well known that the Picard group of each component of $\mathcal{G} r_{G}$ is isomorphic to $\mathbb{Z}$. Let $\mathcal{L}_{\text {det }}$ denote the line bundle on $\mathcal{G} r_{G}$ whose restriction to each component is the positive generator of the Picard group.

4.1. Equivariant line bundles. Fix a representation $V$ of $G$. The homomorphism $\phi: G \rightarrow \mathrm{GL}(V)$ induces a morphism of ind-schemes $\mathcal{G} r_{\phi}: \mathcal{G} r_{G} \rightarrow \mathcal{G} r_{\mathrm{GL}(V)}$ over $\mathbb{C}$. We identify $\mathcal{G} r_{\mathrm{GL}(V)}$ with the set of $\mathcal{O}$-lattices in $V \otimes F$ in the usual way. Fix a lattice $\Lambda_{1} \in \mathcal{G} r_{\mathrm{GL}(V)}$. Define a line bundle $\mathcal{L}_{\Lambda_{1}}$ whose value at a point $\Lambda \in \mathcal{G} r_{\mathrm{GL}(V)}$ is the line

$$
\operatorname{det}\left(\Lambda: \Lambda_{1}\right):=\operatorname{det}\left(\Lambda / \Lambda \cap \Lambda_{1}\right) \otimes \operatorname{det}\left(\Lambda_{1} / \Lambda \cap \Lambda_{1}\right)^{\otimes-1}
$$


where $\operatorname{det}(-)$ means taking the top exterior power of a finite dimensional $\mathbb{C}$-vector space. Let $\mathcal{L}_{\Lambda_{1}}^{\phi}=\mathcal{G} r_{\phi}^{*} \mathcal{L}_{\Lambda_{1}}$ be the pull-back line bundle on $\mathcal{G} r_{G}$.

Let $G(F)_{\Lambda_{1}}$ be the stabilizer of the lattice $\Lambda_{1}$ in $G(F)$ (via the action $\left.\phi\right)$. Then $\mathcal{L}_{\Lambda_{1}}^{\phi}$ has a natural $G(F)_{\Lambda_{1}}$-equivariant structure: for each $g \in G(F)_{\Lambda_{1}}$, we identify $\operatorname{det}\left(\mathcal{G} r_{\phi}(x): \Lambda_{1}\right)$ and $\operatorname{det}\left(\mathcal{G} r_{\phi}(g x): \Lambda_{1}\right)=\operatorname{det}\left(\mathcal{G} r_{\phi}(g x): g \Lambda_{1}\right)$ via the action of $g$. Let $H \subset G(F)_{\Lambda_{1}}$ be an algebraic subgroup. Let $x \in \mathcal{G} r_{G}$ and $H_{x}$ be the stabilizer of $x$ in $H$, so that we can identify the $H$-orbit through $x$ with the homogeneous space $H / H_{x}$. Via the induction functor, we have an identification

$$
\operatorname{Pic}^{H}(H \cdot x)=\operatorname{Pic}^{H}\left(H / H_{x}\right)=\mathbb{X}^{\bullet}\left(H_{x}\right) .
$$

It is easy to see that the image of $\left.\mathcal{L}_{\Lambda_{1}}^{\phi}\right|_{H \cdot x} \in \operatorname{Pic}^{H}(H \cdot x)$ in $\mathbb{X}^{\bullet}\left(H_{x}\right)$ is the character given by the action of $H_{x}$ on the line $\operatorname{det}\left(\mathcal{G} r_{\phi}(x): \Lambda_{1}\right)$ (notice that $H_{x}$ stabilizes both $\mathcal{G} r_{\phi}(x)$ and $\left.\Lambda_{1}\right)$.

We consider the special case where $\Lambda_{0}=V \otimes_{\mathbb{C}} \mathcal{O}$. Since $\Lambda_{0}$ is stabilized by $G(\mathcal{O})$, $\mathcal{L}_{\Lambda_{0}}^{\phi}$ has a natural $G(\mathcal{O})$-equivariant structure. Take $H=G \subset G(\mathcal{O})$ and $x=t^{\lambda}$. Then $H_{x}=P_{\lambda} \subset G$ is a parabolic subgroup containing the maximal torus $T$. We can identify the image of $\mathcal{L}_{\Lambda_{0}}^{\phi}$ in $\mathbb{X}^{\bullet}\left(P_{\lambda}\right) \subset \mathbb{X}^{\bullet}(T)$ as the character of the action of $T$ on $\operatorname{det}\left(t^{\lambda} \Lambda_{0}: \Lambda_{0}\right)$, which is

$$
-\sum_{\chi^{\vee} \in \operatorname{weight}(V)} \operatorname{dim} V_{\chi^{\vee}} \cdot\left\langle\chi^{\vee}, \lambda\right\rangle \chi^{\vee}
$$

We can further specialize to the case $H=H_{x}=T$, then the element in (4.1) is also the restriction of $c_{1}^{T}\left(\mathcal{L}_{\Lambda_{0}}^{\phi}\right)$ to the point $t^{\lambda}$, if we identify $\mathbb{X}^{\bullet}(T)$ with $H_{T}^{2}(\mathrm{pt})$.

4.2. Lemma (See also [KNR94, Section 5] and [So00, Lemma 10.6.1]). We have $\mathcal{L}_{\Lambda_{1}}^{\phi}=\mathcal{L}_{\text {det }}^{\otimes d_{V}} \in \operatorname{Pic}\left(\mathcal{G} r_{G}\right)$ where

$$
d_{V}=\frac{1}{2} \sum_{\chi^{\vee} \in \text { weight }(V)} \operatorname{dim} V_{\chi^{\vee}} \cdot\left\langle\chi^{\vee}, \theta\right\rangle^{2} .
$$

Here $\theta$ stands for the coroot corresponding to the highest root $\theta^{\vee}$ of $G$.

Proof. For $i \in \pi_{0}\left(\mathcal{G} r_{G}\right)$, let $\mathcal{G} r_{G}^{i}$ denote the corresponding connected component. We first claim that the degree of $\mathcal{L}_{\Lambda_{1}}^{\phi}$ on the generating cycle of $H_{2}\left(\mathcal{G} r_{G}^{i}\right)(\cong \mathbb{Z})$ is independent of the component $\mathcal{G} r_{G}^{i}$. In fact, for any $g \in G(F)$, by choosing a trivialization of the line $\operatorname{det}\left(g \Lambda_{1}: \Lambda_{1}\right)$, we get an isomorphism of line bundles $g^{*} \mathcal{L}_{\Lambda_{1}}^{\phi} \cong \mathcal{L}_{\Lambda_{1}}^{\phi}$. Since $G(F)$ transitively permutes the components of $\mathcal{G} r_{G}$, our assertion follows. In the following we concentrate on the neutral component $\mathcal{G} r_{G}^{0}$.

Let $I \subset G(\mathcal{O})$ be the Iwahori subgroup of $G(F)$ containing $B$. The only 1dimensional $I$-orbit in $\mathcal{G} r_{G}^{0}$ is $I t^{-\theta}$, and the fundamental class of its closure generates $H_{2}\left(\mathcal{G} r_{G}^{0}\right)$. Therefore the number $d_{V}$ in question is the degree of $\mathcal{L}_{\Lambda_{1}}^{\phi}$ on the closure of $I t^{-\theta}$.

Fix an $\mathfrak{s l}(2)$-triple $\left(x_{\theta^{\vee}}, \theta, x_{-\theta^{\vee}}\right)$ where $x_{ \pm \theta^{\vee}}$ belongs to the root space $\mathfrak{g}_{\theta^{\vee}}$. Let $\phi_{0}: \mathrm{SL}(2) \rightarrow G(F)$ be the homomorphism which sends the standard triple $(e, h, f)$ to $\left(t^{-1} x_{\theta \vee}, \theta, t x_{-\theta \vee}\right)$. Then the closure of $I t^{-\theta}$ in $\mathcal{G} r_{G}$ is the $\phi_{0}(\mathrm{SL}(2))$-orbit through $t^{-\theta}$, and the stabilizer of $t^{-\theta}$ is the upper triangular matrices $B_{2} \subset \operatorname{SL}(2)$. To compute the degree of $\mathcal{L}_{\Lambda_{1}}^{\phi}$ on this orbit, we will choose a lattice $\Lambda_{1}$ which is stable under the SL(2)-action and calculate the character of $B_{2}$ acting on $\operatorname{det}\left(t^{-\theta} \Lambda_{0}: \Lambda_{1}\right)$. 
Let $V=\bigoplus_{n} V_{n}$ be the grading according to the action of $\theta: V_{n}$ is the direct sum of weight spaces $V_{\chi^{\vee}}$ such that $\left\langle\chi^{\vee}, \theta\right\rangle=n$. Then the lattice $\Lambda_{1}=$ $\bigoplus_{n} t^{-[n / 2]} \mathcal{O} \otimes_{\mathbb{C}} V_{n}$ is stable under the $\mathrm{SL}(2)$-action. On the other hand, the lattice $t^{-\theta} \Lambda_{0}=\bigoplus_{n} t^{-n} \mathcal{O} \otimes_{\mathbb{C}} V_{n}$. Therefore, the action of $B_{2}$ on $\operatorname{det}\left(t^{-\theta} \Lambda_{0}: \Lambda_{1}\right)$ is via a character whose pairing with $\theta$ is:

$$
\sum_{n} n\left(n-\left[\frac{n}{2}\right]\right) \operatorname{dim} V_{n}=\sum_{n} \frac{n^{2}}{2} \operatorname{dim} V_{n}=\frac{1}{2} \sum_{\chi \in \operatorname{weight}(V)} \operatorname{dim} V_{\chi^{\vee}} \cdot\left\langle\chi^{\vee}, \theta\right\rangle^{2} .
$$

Here the first equality follows from the fact that $\operatorname{dim} V_{n}=\operatorname{dim} V_{-n}$. As a wellknown fact for line bundles on $\mathbb{P}^{1}=\mathrm{SL}(2) / B_{2}$, the number in (4.2) is the degree of restriction of the line bundle $\mathcal{L}_{\Lambda_{1}}^{\phi}$ to the $\mathrm{SL}(2)$-orbit through $t^{-\theta}$, hence the degree of $\mathcal{L}_{\Lambda_{1}}^{\phi}$ on the generating cycle of $H_{2}\left(\mathcal{G} r_{G}^{0}\right)$ (here $\Lambda_{1}$ can be any reference lattice, because different choices of $\Lambda_{1}$ give rise to isomorphic line bundles).

4.3. Remark. The lemma shows that some power of $\mathcal{L}_{\text {det }}$ admits a natural $G(\mathcal{O})$ equivariant structure (the $G(\mathcal{O})$-equivariant structure is necessarily unique if $G$ is almost simple). However, $\mathcal{L}_{\text {det }}$ itself may not have a $G(\mathcal{O})$-equivariant structure. Assume that under the isomorphism $\operatorname{Pic}\left(\mathcal{G} r_{G}^{0}\right) \cong \mathbb{Z}$, the image of the natural forgetful map $\operatorname{Pic}^{T}\left(\mathcal{G} r_{G}^{0}\right) \rightarrow \operatorname{Pic}\left(\mathcal{G} r_{G}^{0}\right)$ is $n \mathbb{Z} \subset \mathbb{Z}$. Then the number $n$ coincides with the number $n_{G}$ which will be introduced in Remark 5.8 .

\section{The Regular element $e^{T}$}

We continue using the notation introduced in the previous section. Since $\mathcal{L}_{\Lambda_{0}}^{\phi} \cong$ $\mathcal{L}_{\text {det }}^{\otimes d_{V}}$ admits a canonical $G$-equivariant structure (hence a canonical $T$-equivariant structure), we can take its equivariant Chern class $c_{1}^{T}\left(\mathcal{L}_{\Lambda_{0}}^{\phi}\right) \in H_{T}^{2}\left(\mathcal{G} r_{G}\right)$.

5.1. Lemma. The element $c_{1}^{T}\left(\mathcal{L}_{\Lambda_{0}}^{\phi}\right)$ is primitive in the Hopf algebra $H_{T}^{*}\left(\mathcal{G} r_{G}\right)$.

Proof. Let $m_{K}: \Omega K \times \Omega K \rightarrow \Omega K$ be the multiplication. Then the $T \cap K$ equivariant line bundle $m_{K}^{*}\left(\mathcal{L}_{\Lambda_{0}}^{\phi}\right)$ restricts to $\mathcal{L}_{\Lambda_{0}}^{\phi}$ on both $\Omega K \times\{*\}$ and $\{*\} \times \Omega K$ $(\{*\}$ is the unit element in $\Omega K)$. This forces $c_{1}^{T}\left(\mathcal{L}_{\Lambda_{0}}^{\phi}\right)$ to be primitive. Therefore, $c_{1}^{T}$ is also primitive.

\subsection{Corollary. The element}

$$
c_{1}^{T}=\frac{1}{d_{V}} c_{1}^{T}\left(\mathcal{L}_{\Lambda_{0}}^{\phi}\right) \in H_{T}^{2}\left(\mathcal{G} r_{G}, \mathbb{Q}\right)
$$

is independent of the choice of the representation $\phi: G \rightarrow \mathrm{GL}(V)$.

Proof. For different choices of $(\phi, V)$, the classes $\frac{1}{d_{V}} c_{1}^{T}\left(\mathcal{L}_{\Lambda_{0}}^{\phi}\right)$ have the same image $c_{1}\left(\mathcal{L}_{\text {det }}\right)$ in $H^{2}\left(\mathcal{G} r_{G}\right)$. However, there can be at most one primitive element in $H_{T}^{2}\left(\mathcal{G} r_{G}, \mathbb{Q}\right)$ with a given image in $H^{2}\left(\mathcal{G} r_{G}, \mathbb{Q}\right)$.

5.3. The element $e^{T}$. For each object $\mathcal{F} \in \mathcal{P}_{\mathbb{Q}}$, cup product with $c_{1}^{T}$ induces a functorial map

$$
\cup c_{1}^{T}: H_{T}^{*}(\mathcal{F}) \rightarrow H_{T}^{*+2}(\mathcal{F})
$$


By Proposition 2.7, the fact that $c_{1}^{T}$ is primitive implies that for any $\mathcal{F}_{1}, \mathcal{F}_{2} \in \mathcal{P}_{\mathbb{Q}}$, there is a functorial commutative diagram

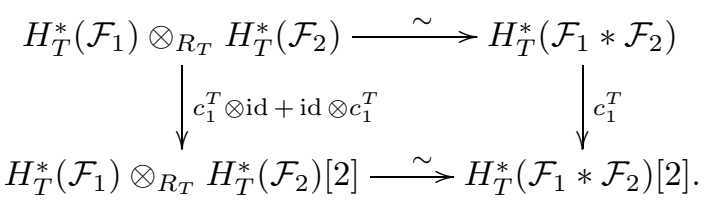

In general, let $c$ is an endomorphism of the functor $\omega_{R}: \operatorname{Rep}\left(G^{\vee}\right) \rightarrow \operatorname{Mod}(R)$ (the composition of the forgetful functor with the tensor product $\otimes R$ ) satisfying $c_{V_{1} \otimes V_{2}}=c_{V_{1}} \otimes \mathrm{id}_{V_{2}}+\mathrm{id}_{V_{1}} \otimes c_{V_{2}}$, then $c$ determines an element $e \in \mathfrak{g}^{\vee} \otimes R$. In fact, Consider the fiber functor $\omega_{R, \epsilon}: \operatorname{Rep}\left(G^{\vee}\right) \rightarrow \operatorname{Mod}\left(R[\epsilon] / \epsilon^{2}\right)$ sending $V \mapsto$ $V \otimes R[\epsilon] / \epsilon^{2}=V \otimes R \oplus V \otimes R \epsilon$. The maps

$$
\left(\begin{array}{cc}
\mathrm{id}_{V} & 0 \\
c_{V} & \mathrm{id}_{V}
\end{array}\right): V \otimes R \oplus V \otimes R \epsilon \rightarrow V \otimes R \oplus V \otimes R \epsilon
$$

give a tensor automorphism of $\omega_{R, \epsilon}$, hence an element $e \in G^{\vee}\left(R[\epsilon] / \epsilon^{2}\right)$. Since the induced tensor automorphism of $\omega_{R}$ (via $R[\epsilon] / \epsilon^{2} \rightarrow R$ ) is the identity, $e$ lies in the kernel of the reduction map $G^{\vee}\left(R[\epsilon] / \epsilon^{2}\right) \rightarrow G^{\vee}(R)$, which is $\mathfrak{g}^{\vee} \otimes R$.

Applying the above discussion to the functor $H_{T}^{*}: \mathcal{P} \rightarrow \operatorname{Mod}\left(R_{T} \otimes \mathbb{Q}\right.$ ) (which can be identified with the functor $\omega_{R_{T} \otimes \mathbb{Q}}: \operatorname{Rep}\left(G^{\vee}\right) \rightarrow \operatorname{Mod}\left(R_{T} \otimes \mathbb{Q}\right)$ by Lemma 2.2) and $c=c_{1}^{T}$, we conclude that $c_{1}^{T}$ gives an element $e^{T} \in \mathfrak{g}^{\vee} \otimes R_{T} \otimes \mathbb{Q}$.

5.4. Remark. More generally, suppose $\omega^{\prime}: \operatorname{Rep}\left(G^{\vee}\right) \rightarrow \operatorname{Mod}(R)$ is a fiber functor and $c^{\prime}$ is an endomorphism of $\omega^{\prime}$ satisfying $c_{V_{1} \otimes V_{2}}^{\prime}=c_{V_{1}}^{\prime} \otimes \operatorname{id}_{\omega^{\prime}\left(V_{2}\right)}+\operatorname{id}_{\omega^{\prime}\left(V_{1}\right)} \otimes c_{V_{2}}^{\prime}$. Then $\omega^{\prime}$ determines a $G^{\vee}$-torsor $\mathcal{E}$ over Spec $R$. Let $\delta: \operatorname{Spec} R[\epsilon] / \epsilon^{2} \rightarrow \operatorname{Spec} R$ be the structure map and let $\delta^{*} \mathcal{E}$ be the pull-back $G^{\vee}$-torsor to Spec $R[\epsilon] / \epsilon^{2}$. Then the same construction as in (5.1) determines an automorphism of $\delta^{*} \mathcal{E}$ whose restriction to the closed subscheme $\operatorname{Spec} R \hookrightarrow \operatorname{Spec} R[\epsilon] / \epsilon^{2}$ is the identity. Spelling these out, we see that $c^{\prime}$ determines a Higgs field of the $G^{\vee}$-torsor $\mathcal{E}$, i.e., a global section $e^{\prime} \in \Gamma(\operatorname{Spec} R, \operatorname{Ad}(\mathcal{E}))$ where $\operatorname{Ad}(\mathcal{E})=\mathcal{E} \stackrel{G^{\vee}}{\times} \mathfrak{g}^{\vee}$ is the adjoint bundle. In other words, the pair $\left(\omega^{\prime}, c^{\prime}\right)$ determines an $R$-point $\left(\mathcal{E}, e^{\prime}\right)$ of the stack $\left[\mathfrak{g}^{\vee} / G^{\vee}\right]$.

Applying the above discussion to the functor $H_{G}^{*}(-)$ (which determines the torsor $\mathcal{E}$ by Remark 2.5) and $c_{1}^{G}(\mathcal{L})$ for any $G$-equivariant line bundle $\mathcal{L}$ on $\mathcal{G} r_{G}$, we get a commutative diagram:

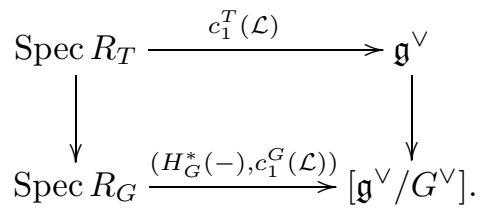

In the remaining part of this section, we determine the element $e^{T}$ explicitly.

We give an (even) grading on $\mathfrak{g}^{\vee}=\bigoplus_{n \in \mathbb{Z}} \mathfrak{g}_{2 n}^{\vee}$ according to the action of $\mathbb{G}_{m}$ via the conjugation of $2 \rho^{\vee}: \mathbb{G}_{m} \rightarrow T^{\vee}$ :

$$
\mathfrak{g}_{2 n}^{\vee}=\bigoplus_{\alpha \in \Phi,\left\langle 2 \rho^{\vee}, \alpha\right\rangle=2 n} \mathfrak{g}_{\alpha}^{\vee}
$$

We give $\mathfrak{g}^{\vee} \otimes R_{T}$ the tensor product (even) grading. The fact that $\cup c_{1}^{T}$ increases the cohomological degree by 2 implies that $e^{T}$ is homogeneous of degree 2 in $\mathfrak{g}^{\vee} \otimes R_{T}$, 
i.e.,

$$
e^{T} \in \bigoplus_{n \in \mathbb{Z}} \mathfrak{g}_{2(1-n)}^{\vee} \otimes \operatorname{Sym}_{n}\left(\mathbb{X}^{\bullet}(T)\right) \otimes \mathbb{Q}
$$

\subsection{Lemma.}

$$
e^{T} \in \mathfrak{g}_{2}^{\vee} \otimes \mathbb{Q} \oplus \mathfrak{t}^{\vee} \otimes \mathbb{X}^{\bullet}(T) \otimes \mathbb{Q} .
$$

Proof. The action of $\cup c_{1}^{T}$ preserves the $T$-equivariant MV-filtration on $H_{T}^{*}(\mathcal{F})$ which gives the canonical $B^{\vee}$-reduction of the trivial $G^{\vee}$-torsor $\left.\mathcal{E}\right|_{\text {Spec } R_{T}}$ (see Remark 2.5). Hence, $e^{T}$ is an $R_{T} \otimes \mathbb{Q}$-valued element of the Lie algebra $\mathfrak{b}^{\vee}$. Since $e^{T}$ is homogeneous of degree 2 in $\mathfrak{b}^{\vee} \otimes R_{T} \otimes \mathbb{Q}$, it must take the form (5.2).

Write $e^{T}=e+h$ where $e \in \mathfrak{g}_{2}^{\vee} \otimes \mathbb{Q}$ and $h \in \mathfrak{t}^{\vee} \otimes \mathbb{X} \bullet(T) \otimes \mathbb{Q}$.

We first calculate $e$. Clearly, $e$ is obtained from the endomorphism of the functor $H^{*}: \mathcal{P} \rightarrow \operatorname{Mod}(\mathbb{Z})$ given by $\cup c_{1}\left(\mathcal{L}_{\text {det }}\right)$, the ordinary Chern class of $\mathcal{L}_{\text {det }}$. In particular, $e \in \mathfrak{g}^{\vee}$. For each simple root $\alpha_{i}$ of $\mathfrak{g}^{\vee}$, let $x_{i}$ be the element of a Chevalley basis of $\mathfrak{g}^{\vee}$ in the root space $\mathfrak{g}_{\alpha_{i}}^{\vee}$. Then $e$ is an integral combination of the $x_{i}$ 's.

5.6. Proposition. Up to changing the Chevalley basis elements $\left\{x_{i}\right\}$ by signs, we have

$$
e=\sum_{i}\left|\alpha_{i}\right|^{2} x_{i}
$$

where ||$^{2}$ is a $W$-invariant quadratic form on $\mathbb{X}_{\bullet}(T)$ normalized so that short coroots (of $G$ ) have length one (hence $\left|\alpha_{i}\right|^{2}=1,2$ or 3 ).

Proof. Let $\mathcal{I}_{*}^{\lambda}$ be the perverse sheaf ${ }^{p} j_{*}^{\lambda} \mathbb{Z}\left[\left\langle 2 \rho^{\vee}, \lambda\right\rangle\right]$ where $j^{\lambda}: \mathcal{G} r_{\lambda} \rightarrow \mathcal{G} r_{G}$ is the inclusion. We first claim that the restriction map $H^{i-\left\langle 2 \rho^{\vee}, \lambda\right\rangle}\left(\mathcal{I}_{*}^{\lambda}\right) \rightarrow H^{i}\left(\mathcal{G} r_{\lambda}\right)$ is an isomorphism for $i \leq 2$. In fact, the cone $\mathcal{F}$ of $\mathcal{I}_{*}^{\lambda} \rightarrow j_{*}^{\lambda} \mathbb{Z}\left[\left\langle 2 \rho^{\vee}, \lambda\right\rangle\right]$ has perverse degree $\geq 1$, and is supported on $\mathcal{G} r_{<\lambda}=\mathcal{G} r_{\leq \lambda}-\mathcal{G} r_{\lambda}$, which has dimension $\leq\left\langle 2 \rho^{\vee}, \lambda\right\rangle-2$. Therefore, $H^{i}(\mathcal{F})$ vanishes for $i<-\operatorname{dim} \mathcal{G} r_{<\lambda}+1 \leq-\left\langle 2 \rho^{\vee}, \lambda\right\rangle+3$. This implies our assertion.

Now we prove the proposition. Without changing $e$ and $\mathfrak{g}^{\vee}$, we can assume that $G$ is of adjoint type. Write $e=\sum_{i} n_{i} x_{i}$ for some integers $n_{i}$. For each simple coroot $\alpha_{i}$ of $G$, let $\lambda_{i}$ be the corresponding fundamental coweight. By MV07, Proposition 13.1], the $G^{\vee}$-module $H^{*}\left(\mathcal{I}_{*}^{-w_{0}\left(\lambda_{i}\right)}\right)$ is the Schur module with highest weight $-w_{0}\left(\lambda_{i}\right)$, where $w_{0}$ stands for the element of longest length in the Weyl group of $G$. Therefore, the Lie algebra element $x_{i}$ sends the lowest weight vector $v_{\text {low }}$ (with weight $-\lambda_{i}$ ) to a generator of the rank 1 weight space of weight $-\lambda_{i}+\alpha_{i}$. For $j \neq i$, clearly $x_{j} \cdot v_{\text {low }}=0$. Therefore, $e \cdot v_{\text {low }}$ is $n_{i}$-times a generator of the rank 1 weight space of weight $-\lambda_{i}+\alpha_{i}$.

Translating to geometry, this means that the action of $c_{1}\left(\mathcal{L}_{\text {det }}\right)$ sends the fundamental class $\left[\mathcal{G} r_{-\lambda_{i}}\right] \in H^{-\left\langle 2 \rho^{\vee},-w_{0}\left(\lambda_{i}\right)\right\rangle}\left(\mathcal{I}_{*}^{-w_{0}\left(\lambda_{i}\right)}\right)$ to $n_{i}$-times a generator of $H^{2-\left\langle 2 \rho^{\vee},-w_{0}\left(\lambda_{i}\right)\right\rangle}\left(\mathcal{I}_{*}^{-w_{0}\left(\lambda_{i}\right)}\right)$ (which is a free $\mathbb{Z}$-module of rank one). By the discussion at the beginning of the proof, we can identify $H^{i-\left\langle 2 \rho^{\vee},-w_{0}\left(\lambda_{i}\right)\right\rangle}\left(\mathcal{I}_{*}^{-w_{0}\left(\lambda_{i}\right)}\right)$ with $H^{i}\left(\mathcal{G} r_{-\lambda_{i}}\right)$. Hence $c_{1}\left(\mathcal{L}_{\text {det }}\right)$ sends $1 \in H^{0}\left(\mathcal{G} r_{-\lambda_{i}}\right)$ to $n_{i}$-times a generator of $H^{2}\left(\mathcal{G} r_{-\lambda_{i}}\right)$ (which is unique up to sign). To calculate this number $n_{i}$, it suffices to calculate the class $\left.c_{1}\left(\mathcal{L}_{\text {det }}\right)\right|_{\mathcal{G} r_{-\lambda_{i}}} \in H^{2}\left(\mathcal{G} r_{-\lambda_{i}}\right)$, or its further restriction to $H^{2}\left(G \cdot t^{-\lambda_{i}}\right)$ (because the inclusion $G \cdot t^{-\lambda_{i}} \hookrightarrow \mathcal{G} r_{-\lambda_{i}}$ is a homotopy equivalence).

Now the restriction of $c_{1}\left(\mathcal{L}_{\mathrm{det}}\right)$ to $G \cdot t^{-\lambda_{i}}$ is $n_{i}$-times the generator of $H^{2}\left(G \cdot t^{-\lambda_{i}}\right)$, which is a free $\mathbb{Z}$-module of rank 1 . Let $\phi_{i}: \operatorname{SL}(2) \rightarrow G$ be the homomorphism 
corresponding to the coroot $\alpha_{i}$ of $G$. Then $\phi_{i}(\mathrm{SL}(2)) \cdot t^{-\lambda_{i}}=\mathrm{SL}(2) / B_{2} \cong \mathbb{P}^{1}$ is the generating 2 -cycle of the partial flag variety $G \cdot t^{-\lambda_{i}}$ (by the Bruhat decomposition), and $n_{i}$ equals the degree of the restriction of $\mathcal{L}_{\text {det }}$ to $\phi_{i}(\mathrm{SL}(2)) \cdot t^{-\lambda_{i}}$.

Let Ad : $G \rightarrow G L(\mathfrak{g})$ denote the adjoint representation of $G$. The degree of $\mathcal{L}_{\Lambda_{0}}^{\mathrm{Ad}}$ on $\phi_{i}(\mathrm{SL}(2)) \cdot t^{-\lambda_{i}} \cong \mathbb{P}^{1}$ is the same as the pairing of $\left.c_{1}^{T}\left(\mathcal{L}_{\Lambda_{0}}^{\mathrm{Ad}}\right)\right|_{t^{-\lambda_{i}}} \in H_{T}^{2}\left(\left\{t^{-\lambda_{i}}\right\}\right)=$ $\mathbb{X}^{\bullet}(T)$ with $\alpha_{i}^{\vee}$. By (4.1), this degree equals

$$
-\sum_{\alpha^{\vee} \in \Phi^{\vee}}\left\langle\alpha^{\vee},-\lambda_{i}\right\rangle\left\langle\alpha^{\vee}, \alpha_{i}\right\rangle=\left(\lambda_{i}, \alpha_{i}\right)_{\mathrm{Kil}} .
$$

By Lemma 4.2, $c_{1}\left(\mathcal{L}_{\Lambda_{0}}^{\mathrm{Ad}}\right)=d_{\mathrm{Ad}} c_{1}\left(\mathcal{L}_{\mathrm{det}}\right)=\frac{1}{2}(\theta, \theta)_{\mathrm{Kil}} c_{1}\left(\mathcal{L}_{\mathrm{det}}\right)$, therefore the degree of $\mathcal{L}_{\text {det }}$ on $\phi_{i}(\operatorname{SL}(2)) \cdot t^{-\lambda_{i}}$ is

$$
n_{i}=\frac{2\left(\lambda_{i}, \alpha_{i}\right)_{\mathrm{Kil}}}{(\theta, \theta)_{\mathrm{Kil}}}=\frac{\left(\alpha_{i}, \alpha_{i}\right)_{\mathrm{Kil}}}{(\theta, \theta)_{\mathrm{Kil}}} .
$$

Since $\theta$ is a short coroot, we conclude that $n_{i}=\left|\alpha_{i}\right|^{2}$.

Next we determine the element $h \in \mathfrak{t}^{\vee} \otimes \mathbb{X} \bullet(T) \otimes \mathbb{Q}$.

5.7. Proposition. Identifying $\mathfrak{t}^{\vee}$ with $\mathbb{X} \bullet(T)$ and $\mathfrak{t}^{\vee} \otimes \mathbb{X}^{\bullet}(T)$ with the dual of $\mathbb{X}_{\bullet}(T) \otimes \mathbb{X}_{\bullet}(T)$, the element $h$ is the $(\mathbb{Q}$-valued) symmetric bilinear form

$$
h(\lambda, \mu)=\frac{-2(\lambda, \mu)_{\mathrm{Kil}}}{(\theta, \theta)_{\mathrm{Kil}}} .
$$

Proof. Write $h=\sum h_{i} \otimes g_{i}$, where $h_{i} \in \mathfrak{t}^{\vee}, g_{i} \in \mathbb{X} \bullet(T)$. Since the cup product with $c_{1}^{T}$ preserves the $T$-equivariant MV-filtration, there is an induced action $\overline{e^{T}}$ on the associated graded pieces $H_{T, c}^{*}\left(S_{\lambda},-\right)$. The element $h$ is given by the action $\overline{e^{T}}$. More precisely, for $\lambda \in \mathbb{X}_{\bullet}(T)$, the action of $\overline{e^{T}}$ on the $\lambda$-weight subspace is given by the multiplication of $\sum\left\langle h_{i}, \lambda\right\rangle g_{i}$. Translating back to geometry, this means that the cup product of $c_{1}^{T}$ on $H_{T, c}^{*}\left(S_{\lambda},-\right)$ should be the multiplication by $\sum\left\langle h_{i}, \lambda\right\rangle g_{i}$ (where as before, $g_{i} \in \mathbb{X}^{\bullet}(T) \cong H_{T}^{2}(\mathrm{pt})$ ). On the other hand, $c_{1}^{T}$ acts on $H_{T, c}^{*}\left(S_{\lambda},-\right)$ via its restriction to $S_{\lambda}$. Since $\left\{t^{\lambda}\right\} \hookrightarrow S_{\lambda}$ is a $T$-equivariant homotopy equivalence, the action of $c_{1}^{T}$ on $H_{T, c}^{*}\left(S_{\lambda},-\right)$ is given by the multiplication of $i_{\lambda}^{*}\left(c_{1}^{T}\right) \in H_{T}^{2}\left(\left\{t^{\lambda}\right\}, \mathbb{Q}\right)=\mathbb{X} \cdot(T) \otimes \mathbb{Q}$.

Applying (4.1) to the line bundle $\mathcal{L}_{\Lambda_{0}}^{\mathrm{Ad}}$ associated with the adjoint representation Ad of $G$, and using Lemma 4.2, we see that the restriction of $c_{1}^{T}$ to $H_{T}^{2}\left(\left\{t^{\lambda}\right\}\right)$ is given by:

$$
i_{\lambda}^{*}\left(c_{1}^{T}\right)=\frac{-2}{(\theta, \theta)_{\mathrm{Kil}}} \sum_{\alpha^{\vee} \in \Phi^{\vee}}\left\langle\alpha^{\vee}, \lambda\right\rangle \alpha^{\vee} .
$$

Therefore,

$$
h=\frac{-2}{(\theta, \theta)_{\mathrm{Kil}}} \sum_{\alpha^{\vee} \in \Phi^{\vee}} \alpha^{\vee} \otimes \alpha^{\vee} .
$$

If we view $h$ as a bilinear form on $\mathbb{X}_{\bullet}(T)$, then for any $\lambda, \mu \in \mathbb{X}_{\bullet}(T)$,

$$
h(\lambda, \mu)=\frac{-2 \sum_{\alpha^{\vee} \in \Phi^{\vee}}\left\langle\alpha^{\vee}, \lambda\right\rangle\left\langle\alpha^{\vee}, \mu\right\rangle}{(\theta, \theta)_{\mathrm{Kil}}}=\frac{-2(\lambda, \mu)_{\mathrm{Kil}}}{(\theta, \theta)_{\mathrm{Kil}}} .
$$


5.8. Remark. Let $n_{G}$ be the least positive integer such that $n_{G} h \in \mathfrak{t}^{\vee} \otimes \mathbb{X}^{\bullet}(T)$ (or equivalently, such that $\left.n_{G} e^{T} \in \mathfrak{g}^{\vee} \otimes R_{T}\right)$. Clearly, we have $n_{G}=n_{G^{\text {der }}}$. On the other hand, for $G$ is almost simple, this number can be found in [So00, Table C], in which $n_{G}$ is denoted by $\ell_{b}$. We reproduce these numbers explicitly in the following list:

- $n_{G}=\frac{\# \pi_{1}(G)}{\operatorname{gcd}\left(\# Z(G), \# \pi_{1}(G)\right)}$ if $G$ is of type A;

- $n_{G}=2$ if $G$ is $\mathbf{P S p}_{2 m}$ (for $m$ odd), $\mathbf{S O}_{4 m}^{ \pm}$(for $m$ odd), $\mathbf{P} \mathbf{S O}_{4 m}$, or $\mathbf{P} \mathbf{E}_{7}$;

- $n_{G}=3$ if $G$ is $\mathbf{P E}$;

- $n_{G}=4$ if $G$ is $\mathbf{P S O}_{4 m+2}$;

- $n_{G}=1$ otherwise.

Here $\mathbf{P}$ stands for the adjoint form, and $\mathbf{S O}_{4 m}^{ \pm}$are the two quotients of $\mathbf{S p i n} \mathbf{i n}_{4 m}$ by $\mathbb{Z} / 2$ which are not isomorphic to $\mathbf{S O}_{4 m}$.

\section{Proof of the MAIN RESUlt}

Recall the morphism $\widetilde{\sigma}^{T}: \operatorname{Spec} H_{*}^{T}\left(\mathcal{G} r_{G}\right) \rightarrow B^{\vee} \times \operatorname{Spec} R_{T}$ defined in Proposition $3.3(1)$ and its non-equivariant counterpart $\widetilde{\sigma}: \operatorname{Spec} H_{*}\left(\mathcal{G} r_{G}\right) \rightarrow B^{\vee}$. It is clear that the action of $\sigma_{c a n}$ on the fiber functor $H_{T}^{*}(-) \otimes_{R_{T}} H_{*}^{T}\left(\mathcal{G} r_{G}\right)$ commutes with the cup product action of $c_{1}^{T}$. Therefore, $\widetilde{\sigma}^{T}$ further factors through the homomorphism

$$
\sigma^{T}: \operatorname{Spec} H_{*}^{T}\left(\mathcal{G} r_{G}\right)\left[1 / n_{G}\right] \rightarrow B_{e^{T}}^{\vee},
$$

of group schemes over Spec $R_{T}\left[1 / n_{G}\right]$. Here $B_{e^{T}}^{\vee}$ is the centralizer group scheme of $e^{T} \in \mathfrak{g}^{\vee}\left(R_{T}\left[1 / n_{G}\right]\right)$ in $B^{\vee} \times \operatorname{Spec} R_{T}\left[1 / n_{G}\right]$. Since $e$ is already an element in $\mathfrak{g}^{\vee}$, we also have the homomorphism

$$
\sigma: \operatorname{Spec} H_{*}\left(\mathcal{G} r_{G}\right) \rightarrow Z^{\vee} \times U_{e}^{\vee} \hookrightarrow B_{e}^{\vee}
$$

of group schemes over $\mathbb{Z}$ (the first arrow above follows from Proposition [3.3(4)).

6.1. Theorem. Assume that $G^{\text {der }}$ is almost simple.

(1) The homomorphism $\sigma: \operatorname{Spec}_{*}\left(\mathcal{G}_{G}\right) \rightarrow B_{e}^{\vee}$ is a closed embedding, and is an isomorphism over $\operatorname{Spec} \mathbb{Z}\left[1 / \ell_{G}\right]$, where $\ell_{G}=1,2$ or 3 is the square of the ratio of lengths of the long and short coroots of $G$. In particular, $\operatorname{Spec} H_{*}\left(\mathcal{G} r_{G}\right) \rightarrow Z^{\vee} \times U_{e}^{\vee}$ is also an isomorphism over $\operatorname{Spec} \mathbb{Z}\left[1 / \ell_{G}\right]$.

(2) The homomorphism $\sigma^{T}$ : Spec $H_{*}^{T}\left(\mathcal{G} r_{G}\right)\left[1 / n_{G}\right] \rightarrow B_{e^{T}}^{\vee}$ is a closed embedding, and is an isomorphism over Spec $R_{T}\left[\frac{1}{\ell_{G} n_{G}}\right]$.

Proof. Step I. We first prove that $\sigma^{T}$ and $\sigma$ are closed embeddings, i.e., $\tau^{T}$ : $\mathcal{O}\left(B_{e^{T}}^{\vee}\right) \rightarrow H_{*}^{T}\left(\mathcal{G} r_{G}\right)\left[1 / n_{G}\right]$ and $\tau: \mathcal{O}\left(B_{e}^{\vee}\right) \rightarrow H_{*}\left(\mathcal{G} r_{G}\right)$ are surjective. It suffices to prove the surjectivity of $\tau$ because any homogeneous lifting of a $\mathbb{Z}\left[1 / n_{G}\right]$-basis of $H_{*}\left(\mathcal{G} r_{G}\right)\left[1 / n_{G}\right]$ to $H_{*}^{T}\left(\mathcal{G} r_{G}\right)\left[1 / n_{G}\right]$ generates $H_{*}^{T}\left(\mathcal{G} r_{G}\right)\left[1 / n_{G}\right]$ as an $R_{T}\left[1 / n_{G}\right]$ module, and we can choose these liftings to be in the image of $\tau^{T}$ since $\mathcal{O}\left(B_{e^{T}}^{\vee}\right) \rightarrow$ $\mathcal{O}\left(B_{e}^{\vee}\right)$ is surjective.

Fix a dominant coweight $\lambda$ of $G$. Consider the following diagram:

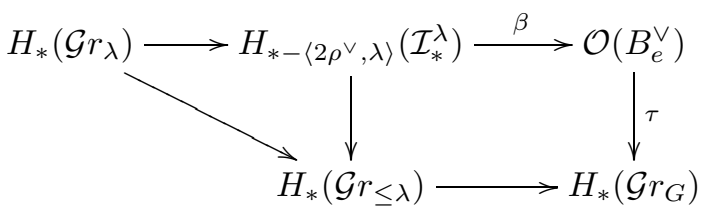


where $H_{*}\left(\mathcal{I}_{*}^{\lambda}\right)$ is the $\mathbb{Z}$-dual of $H^{*}\left(\mathcal{I}_{*}^{\lambda}\right)$. In the following, we describe the various arrows in this diagram and verify its commutativity.

First we concentrate on the triangle in (6.1). We have the natural morphisms between complexes on $\mathcal{G} r_{\leq \lambda}$ :

$$
\mathbb{Z} \rightarrow \mathcal{I}_{*}^{\lambda}\left[-\left\langle 2 \rho^{\vee}, \lambda\right\rangle\right] \rightarrow j_{*}^{\lambda} \mathbb{Z}
$$

Taking duals of their cohomology, we get the triangle in (6.1), which is therefore commutative.

Next we examine the square in (6.1). The map $\beta$ is defined to be the matrix coefficient of the $G^{\vee}$-module $H^{*}\left(\mathcal{I}_{*}^{\lambda}\right)$ with respect to the lowest weight vector

$$
v_{\text {low }}=\left[\mathcal{G} r_{\leq \lambda}\right] \in H^{-\left\langle 2 \rho^{\vee}, \lambda\right\rangle}\left(\mathcal{I}_{*}^{\lambda}\right) .
$$

More precisely, for $u \in H_{*}\left(\mathcal{I}_{*}^{\lambda}\right)$,

$$
\beta(u)(g)=\left\langle u, g \cdot v_{l o w}\right\rangle, \forall g \in B_{e}^{\vee} .
$$

We check that commutativity of the square in (6.1). For $u \in H_{*}\left(\mathcal{I}_{*}^{\lambda}\right)$ and the $H_{*}\left(\mathcal{G} r_{G}\right)$-valued point $\sigma_{c a n} \in B_{e}^{\vee}\left(H_{*}\left(\mathcal{G} r_{G}\right)\right)$, we have

$$
\tau \beta(u)=\beta(u)\left(\sigma_{\text {can }}\right)=\left\langle u, \sigma_{\text {can }} v_{\text {low }}\right\rangle=\sum_{i}\left\langle u, h^{i} \cdot v_{\text {low }}\right\rangle h_{i} \in H_{*}\left(\mathcal{G} r_{G}\right)
$$

where $\left\{h^{i}\right\},\left\{h_{i}\right\}$ are dual bases of $H^{*}\left(\mathcal{G} r_{G}\right)$ and $H_{*}\left(\mathcal{G} r_{G}\right)$. On the other hand, the map $H^{*}\left(\mathcal{G} r_{\leq \lambda}\right) \rightarrow H^{*-\left\langle 2 \rho^{\vee}, \lambda\right\rangle}\left(\mathcal{I}_{*}^{\lambda}\right)$ sends $h \mapsto h \cdot v_{\text {low }}$, hence the adjoint map $H_{*-\left\langle 2 \rho^{\vee}, \lambda\right\rangle}\left(\mathcal{I}_{*}^{\lambda}\right) \stackrel{\rightarrow}{\rightarrow} H_{*}\left(\mathcal{G}_{\leq \lambda}\right)$ sends $u \mapsto \sum_{i}\left\langle u, h^{i} \cdot v_{\text {low }}\right\rangle h_{i}$. Combining with (6.2), we have verified that the square in (6.1) is commutative.

From diagram (6.1), we conclude that the image of $\mathcal{O}\left(B_{e}^{\vee}\right) \rightarrow H_{*}\left(\mathcal{G} r_{G}\right)$ contains the image of $H_{*}\left(G \cdot t^{\lambda}\right)=H_{*}\left(\mathcal{G} r_{\lambda}\right) \rightarrow H_{*}\left(\mathcal{G} r_{G}\right)$ for any $\lambda \in \mathbb{X}_{\bullet}(T)$. When $G$ is of adjoint type, by [B58, Theorem 1], there exists a single $\lambda$ (called the "generating circle" by Bott), such that the image of $H_{*}\left(G \cdot t^{\lambda}\right) \rightarrow H_{*}\left(\mathcal{G} r_{G}\right)$ generates $H_{*}\left(\mathcal{G} r_{G}\right)$ as a $\mathbb{Z}$-algebra. This implies that $\mathcal{O}\left(B_{e}^{\vee}\right) \rightarrow H_{*}\left(\mathcal{G} r_{G}\right)$ is surjective in the case $G$ is adjoint. By Proposition [3.3(4), this means that $\operatorname{Spec} H_{*}\left(\mathcal{G} r_{G}\right)=$ Spec $H_{0}\left(\mathcal{G} r_{G}\right) \otimes H_{*}\left(\mathcal{G} r_{G^{s c}}\right) \rightarrow Z^{\vee} \times U^{\vee}$ is a closed embedding when $G$ is adjoint. Since Spec $H_{0}\left(\mathcal{G} r_{G}\right) \cong Z^{\vee}$, we conclude that $H_{*}\left(\mathcal{G} r_{G^{s c}}\right) \rightarrow U^{\vee}$ is also a closed embedding. For general $G$, using Proposition 3.3(4) again, we conclude that Spec $H_{*}\left(\mathcal{G} r_{G}\right) \rightarrow Z^{\vee} \times U^{\vee}$ is always a closed embedding.

Step II. We prove that $B_{e^{T}}^{\vee}\left[1 / \ell_{G}\right]$ is flat over $R_{T}^{\prime}:=R_{T}\left[\frac{1}{\ell_{G} n_{G}}\right]$. Consider the morphism over $R_{T}^{\prime}$ :

$$
\phi=\operatorname{Ad}(-) e^{T}-e^{T}: B^{\vee} \times \operatorname{Spec} R_{T}^{\prime} \rightarrow \mathfrak{u}^{\vee} \times \operatorname{Spec} R_{T}^{\prime} .
$$

Then $B_{e^{T}}^{\vee}\left[1 / \ell_{G}\right]=\phi^{-1}(0)$, i.e., $B_{e^{T}}^{\vee}\left[1 / \ell_{G}\right]$ is the closed subscheme of the $B^{\vee} \times$ Spec $R_{T}^{\prime}$ (which is smooth over $R_{T}^{\prime}$ ) cut out by $\operatorname{dim} \mathfrak{u}^{\vee}$ equations. Hence the fiber dimensions of $B_{e^{T}}^{\vee} \rightarrow \operatorname{Spec} R_{T}^{\prime}$ are at least $\operatorname{dim} B^{\vee}-\operatorname{dim} \mathfrak{u}^{\vee}=r$, the rank of $G^{\vee}$. If we can show that all the fibers of $B_{e^{T}}^{\vee}\left[1 / \ell_{G}\right] \rightarrow$ Spec $R_{T}^{\prime}$ are $r$-dimensional, then $B_{e^{T}}^{\vee}\left[1 / \ell_{G}\right]$ will be locally a complete intersection over $R_{T}^{\prime}$. Using that all the fibers are of the same dimension again and that $R_{T}^{\prime}$ is regular, we can conclude that $B_{e^{T}}^{\vee}$ is flat over $R_{T}^{\prime}$ (see [M80, 20.D] with obvious modifications). Therefore, it suffices to show that for any closed point $s \in \operatorname{Spec} R_{T}^{\prime}$, the fiber $\left(B_{e}^{\vee}\right)_{s}$ has dimension $r$.

Since $R_{T}$ is graded, Spec $R_{T}$ admits a natural $\mathbb{G}_{m}$-action with fixed point locus $z: \operatorname{Spec} \mathbb{Z} \hookrightarrow \operatorname{Spec} R_{T}$ defined by the augmentation ideal of $R_{T}$. Moreover, this fixed point locus is attracting: for each point $s \in \operatorname{Spec} R_{T}$, let $\eta \in \operatorname{Spec} R_{T}$ be the 
generic point of the $\mathbb{G}_{m}$-orbit of $s$, then the closure of $\eta$ intersects the fixed point locus $z(\operatorname{Spec} \mathbb{Z})$. In other words, $\eta$ specializes to a point $s_{0} \in z(\operatorname{Spec} \mathbb{Z})$ of the same residue characteristic as $s$. Hence $\operatorname{dim}\left(B_{e}^{\vee}\right)_{s}=\operatorname{dim}\left(B_{e}^{\vee}\right)_{\eta} \leq \operatorname{dim}\left(B_{e_{0}}^{\vee}\right)_{s_{0}}$. Therefore, in order to show that all fibers of $B_{e^{T}}^{\vee}\left[1 / \ell_{G}\right] \rightarrow \operatorname{Spec} R_{T}^{\prime}$ have dimension $r$, it suffices to show that all (geometric) fibers of $B_{e}^{\vee}\left[1 / \ell_{G}\right] \rightarrow$ Spec $\mathbb{Z}\left[1 / \ell_{G}\right]$ have dimension $r$.

Let $k$ be an algebraic closure of $\mathbb{F}_{p}$ for some prime $p$ not dividing $\ell_{G}$. We would like to show that $B_{e}^{\vee} \otimes_{\mathbb{Z}} k$ has dimension $r$ over $k$. We base change the situation from $\mathbb{Z}$ to $k$ without changing notation. So in the rest of this paragraph $B_{e}^{\vee}$ is over $k$, etc. By Proposition 5.6, the element $e$ has the form $\sum_{i} n_{i} x_{i}$ where each $n_{i} \neq 0$ in $k$. We can choose $t \in T^{\vee}(k)$ such that $\operatorname{Ad}(t) e=\sum_{i} x_{i}$ (because $\prod \alpha_{i}: T^{\vee}(k) \rightarrow\left(k^{\times}\right)^{r}$ is surjective). Therefore, we only need to treat the element $e_{1}=\sum_{i} x_{i}$. It is well known that $B_{e_{1}}^{\vee}=Z^{\vee} \times U_{e_{1}}^{\vee}$; therefore, it suffices to show that $\operatorname{dim} U_{e_{1}}^{\vee}=r-\operatorname{dim} Z^{\vee}$. This equality follows from the main result of [K87]. This proves that $B_{e^{T}}^{\vee}$ is flat over $R_{T}^{\prime}$.

The above argument also shows that $B_{e}^{\vee}\left[1 / \ell_{G}\right]$ is flat over $\mathbb{Z}\left[1 / \ell_{G}\right]$.

Step III. Now we can finish the proof.

(2) The equivariant version. In view of Step I, it remains to prove that

$$
\mathcal{O}\left(B_{e^{T}}^{\vee}\right)\left[1 / \ell_{G}\right] \rightarrow H_{*}^{T}\left(\mathcal{G} r_{G}\right)\left[\frac{1}{\ell_{G} n_{G}}\right]
$$

is injective. Since both $\mathcal{O}\left(B_{e^{T}}^{\vee}\right)\left[1 / \ell_{G}\right]$ and $H_{*}^{T}\left(\mathcal{G} r_{G}\right)\left[\frac{1}{\ell_{G} n_{G}}\right]$ are flat over $R_{T}^{\prime}$, it suffices to show that $\mathcal{O}\left(B_{e}^{\vee}\right)\left[1 / \ell_{G}\right] \otimes_{R_{T}^{\prime}} Q \rightarrow H_{*}^{T}\left(\mathcal{G} r_{G}\right) \otimes_{R_{T}} Q$ is injective where $Q=\operatorname{Frac}\left(R_{T}\right)$.

Recall from Remark 3.4 that we have a commutative diagram

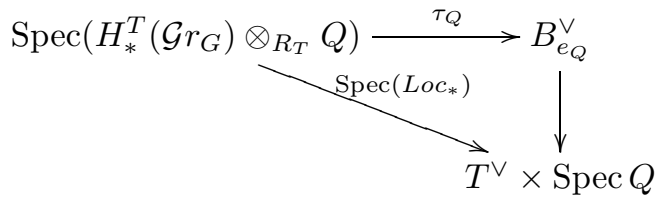

where $e_{Q} \in \mathfrak{b}^{\vee}(Q)$ is the value of $e^{T}$ over the generic point of Spec $R_{T}$. By the equivariant localization theorem, the localization map $L o c_{*}$ in (3.10) is an isomorphism. On the other hand, by Proposition 5.7 $e_{Q}$ is a regular semisimple element in $\mathfrak{b}^{\vee}(Q)$, hence the projection $B_{e_{Q}}^{\vee} \rightarrow T^{\vee} \times \operatorname{Spec} Q$ is also an isomorphism. This shows that the closed embedding $\tau_{Q}$ is in fact an isomorphism, and (2) is proved.

(1) The non-equivariant version. As a consequence of the equivariant version, $\sigma: \operatorname{Spec} H_{*}\left(\mathcal{G} r_{G}\right) \rightarrow B_{e}^{\vee}$ is an isomorphism over $\mathbb{Q}$ (we base change $\sigma^{T}$ to $\operatorname{Spec} \mathbb{Q} \rightarrow \operatorname{Spec} \mathbb{Z} \stackrel{z}{\rightarrow}$ Spec $\left.R_{T}\right)$. Therefore, $\tau \otimes \mathbb{Q}: \mathcal{O}\left(B_{e}^{\vee}\right) \otimes \mathbb{Q} \rightarrow H_{*}\left(\mathcal{G} r_{G}, \mathbb{Q}\right)$ is an isomorphism. Since $\mathcal{O}\left(B_{e}^{\vee}\right)\left[1 / \ell_{G}\right]$ is flat over $\mathbb{Z}\left[1 / \ell_{G}\right]$ by Step II, hence torsion-free, we conclude that $\tau\left[1 / \ell_{G}\right]: \mathcal{O}\left(B_{e}^{\vee}\right)\left[1 / \ell_{G}\right] \rightarrow H_{*}\left(\mathcal{G} r_{G}\right)\left[1 / \ell_{G}\right]$ is injective. Combining this with Step I, (1) is proved.

\subsection{Remark.}

(1) Inverting $\ell_{G}$ in the statement of the above theorem is necessary because $e$ is not regular modulo $\ell_{G}$, hence the fiber of $B_{e}^{\vee}$ over $\mathbb{F}_{\ell_{G}}$ does not have the same dimension as $\operatorname{Spec} H_{*}\left(\mathcal{G} r_{G}, \mathbb{F}_{\ell_{G}}\right)$. However, the proof of the above theorem implies that $\operatorname{Spec} H_{*}\left(\mathcal{G} r_{G}\right)$ is always isomorphic to the Zariski closure in $B_{e}^{\vee}$ of its generic fiber. 
(2) The theorem shows that no primes other than $\ell_{G}$ need to be inverted, even for those "bad primes" of $G$ (i.e., those dividing the coefficients of the highest root in terms of a linear combination of simple roots). For example, let $G$ be the exceptional group $G_{2}$. Then Bott (cf. [B58]) shows that

$$
H_{*}(\Omega K) \cong \mathbb{Z}[u, v, w] /\left(2 v-u^{2}\right) .
$$

On the other hand, one can show (using formulae in [K87]) that

$\mathcal{O}\left(B_{e}^{\vee}\right) \cong \mathbb{Z}[A, C, D, E, F] /\left(2 C-3 A^{2}, 3\left(D-A^{3}\right), 3\left(E+C^{2}-3 A^{4}\right)\right)$.

We find that $H_{*}\left(\Omega K, \mathbb{F}_{2}\right) \cong \mathcal{O}\left(B_{e}^{\vee}\right) \otimes \mathbb{F}_{2}$ although 2 is considered as a bad prime for group $G_{2}$.

6.3. Description of the cohomology. Dually, we have a description of the cohomology of $\mathcal{G} r_{G}$. Recall that if $H$ is an affine group scheme over some commutative ring $k$, the algebra of distributions on $H$ (cf. [J03, §I.7]) is defined as

$$
\operatorname{Dist}(H)=\left\{f: \mathcal{O}(H) \rightarrow k \mid f \text { is } k \text {-linear, } f\left(\mathfrak{m}^{n+1}\right)=0 \text { for some } n\right\} .
$$

Here $\mathfrak{m}$ is the augmented ideal of $\mathcal{O}(H)$. It has a natural algebra structure dual to the coalgebra structure on $\mathcal{O}(H)$. Dist $(H)$ can be also regarded as the algebra of left invariant differential operators on $H$. If, in addition, $H$ is infinitesimally flat (i.e. $\mathcal{O}(H) / \mathfrak{m}^{n+1}$ is flat over $k$ for any $n$ ), then $\operatorname{Dist}(H)$ has a natural structure of a Hopf algebra over $k$.

Now let $H=B_{e}^{\vee}$. It is proved by Springer (cf. [Sp66]) that $B_{e}^{\vee} \otimes \mathbb{F}_{p}$ is smooth if the prime $p$ is good (i.e. not bad) for $G^{\vee}$. This fact, together with the flatness of $B^{\vee}$ over $\mathbb{Z}\left[1 / \ell_{G}\right]$ as shown in the course of proving Theorem 6.1, implies that $B_{e}^{\vee}$ is smooth over $\mathbb{Z}^{\prime}$, where $\mathbb{Z}^{\prime}$ is obtained from $\mathbb{Z}$ by inverting the bad primes of $G^{\vee}$. In particular, $B_{e}^{\vee}$ is infinitesimally flat over $\mathbb{Z}^{\prime}$.

6.4. Corollary. Assume that $G$ is almost simple and simply-connected. Then there is an isomorphism of algebras over $\mathbb{Z}$,

$$
\tau^{*}: H^{*}\left(\mathcal{G} r_{G}\right) \stackrel{\sim}{\rightarrow} \operatorname{Dist}\left(U_{e}^{\vee}\right)
$$

which is an isomorphism of Hopf algebras over $\mathbb{Z}^{\prime}$.

Proof. Observe that $Z^{\vee}$ is trivial in this case, hence $U_{e}^{\vee}=B_{e}^{\vee}$. In the course of proving Theorem 6.1 we have shown that $\tau: \mathcal{O}\left(U^{\vee}\right) \rightarrow H_{*}\left(\mathcal{G} r_{G}\right)$ is surjective, and is an isomorphism up to torsion. Observe that $\operatorname{Dist}\left(U_{e}^{\vee}\right)$ can be identified with the graded dual of $\mathcal{O}\left(U_{e}^{\vee}\right)$ because elements in the augmentation ideal of $\mathcal{O}\left(U_{e}^{\vee}\right)$ all have positive degrees. Taking the graded dual of $\tau$ we get the desired isomorphism $\tau^{*}$ in (6.3).

6.5. Description of the $G$-equivariant homology. In this subsection, we assume that $G$ is almost simple and simply-connected. We now interpret the $G$ equivariant homology $H_{*}^{G}\left(\mathcal{G} r_{G}\right)$ in terms of the regular centralizer group scheme of $G^{\vee}$. Let us recall that a prime $p$ is called a torsion prime of $G$ if $H^{*}(G, \mathbb{Z})$ has $p$-torsion. Let $S_{1}$ be the multiplicative set generated by the torsion primes of $G$, and let $\mathbb{Z}_{S_{1}}$ be the corresponding localization, i.e., the localization away from the torsion primes. Borel (see $\mathrm{B} 53$ ) proved that

$$
R_{G} \otimes \mathbb{Z}_{S_{1}} \stackrel{\sim}{\rightarrow}\left(R_{T} \otimes \mathbb{Z}_{S_{1}}\right)^{W} .
$$

Since $N_{G}(T)$ acts on $\mathcal{G} r_{G}$, it induces an action of $W=N_{G}(T) / T$ on $H_{*}^{T}\left(\mathcal{G} r_{G}\right)$ compatible with the natural $W$-action on $R_{T}$. The natural map $H_{*}^{G}\left(\mathcal{G} r_{G}\right) \rightarrow H_{*}^{T}\left(\mathcal{G} r_{G}\right)$ 
then factors through the $W$-invariants of $H_{*}^{T}\left(\mathcal{G} r_{G}\right)$. Using the isomorphism (6.4), it is easy to show that

$$
H_{*}^{G}\left(\mathcal{G} r_{G}, \mathbb{Z}_{S_{1}}\right) \stackrel{\sim}{\rightarrow} H_{*}^{T}\left(\mathcal{G} r_{G}, \mathbb{Z}_{S_{1}}\right)^{W}
$$

as Hopf algebras over $R_{G} \otimes \mathbb{Z}_{S_{1}}$.

On the other hand, let $S_{2}$ be the multiplicative set generated by bad primes of $G^{\vee}$ and those dividing $n+1$ if $G$ is of type $A_{n}$. Clearly, $\ell_{G}, n_{G} \in S_{2}$, and the result in [B60] implies $S_{1} \subset S_{2}$. Let $\mathbb{Z}_{S_{2}}$ be the localization of $\mathbb{Z}$ with respect to $S_{2}$. Consider the universal centralizer scheme $I^{\vee}$ over $\mathfrak{g}^{\vee}$ (i.e., the fiber of $I^{\vee}$ over $x \in \mathfrak{g}^{\vee}$ is the centralizer $\left.G_{x}^{\vee}\right)$. After inverting primes in $S_{2}$, we have the following identifications:

$$
\left(\mathfrak{g}^{\vee} / / G\right) \times \operatorname{Spec} \mathbb{Z}_{S_{2}} \stackrel{\sim}{\rightarrow}\left(\mathfrak{t}^{\vee} / / W\right) \times \operatorname{Spec} \mathbb{Z}_{S_{2}} \stackrel{\sim}{\rightarrow}\left(\operatorname{Spec} R_{G} \otimes \mathbb{Z}_{S_{2}}\right)
$$

where the latter isomorphism uses the element $f$ in Proposition 5.7. It can be shown that the restriction of $I^{\vee}$ to the regular locus $\left(\mathfrak{g}^{\vee}\right)^{r e g} \times \operatorname{Spec} \mathbb{Z}_{S_{2}}$ descends to a smooth group scheme $J^{\vee}$ over $\operatorname{Spec}\left(R_{G} \otimes \mathbb{Z}_{S_{2}}\right) \cong\left(\mathfrak{g}^{\vee} / / G\right) \times \operatorname{Spec} \mathbb{Z}_{S_{2}}$. In fact, this is essentially proved in [N06] with all primes dividing \#W inverted; but further argument shows that only primes in $S_{2}$ need to be inverted.

6.6. Proposition. There is a natural isomorphism of group schemes over $\operatorname{Spec}\left(R_{G} \otimes \mathbb{Z}_{S_{2}}\right)$ :

$$
\operatorname{Spec} H_{*}^{G}\left(\mathcal{G} r_{G}, \mathbb{Z}_{S_{2}}\right) \stackrel{\sim}{\rightarrow} J^{\vee} .
$$

Sketch of proof. Let $\pi: \operatorname{Spec}\left(R_{T} \otimes \mathbb{Z}_{S_{2}}\right) \rightarrow \operatorname{Spec}\left(R_{G} \otimes \mathbb{Z}_{S_{2}}\right)$, then by the definition of the regular centralizer, we have a closed embedding $\beta: B_{e^{T}}^{\vee} \rightarrow G_{e^{T}}^{\vee}=\pi^{*} J^{\vee}$ (from now on we view $B_{e^{T}}^{\vee}$ as a scheme over $\operatorname{Spec}\left(R_{T} \otimes \mathbb{Z}_{S_{2}}\right)$ ). Using the flatness of $B_{e^{T}}^{\vee}$ and $\pi^{*} J^{\vee}$ over $R_{G} \otimes \mathbb{Z}_{S_{2}}$, it is easy to see that $\beta$ is an isomorphism. Therefore, the coordinate ring $\mathcal{O}\left(J^{\vee}\right)$ can be identified with the $W$-invariants of $\mathcal{O}\left(B_{e^{T}}^{\vee} \stackrel{\sim}{\rightarrow} \mathcal{O}\left(G_{e^{T}}^{\vee}\right)\right.$. Recall from (6.5) that $H_{*}^{G}\left(\mathcal{G} r_{G}, \mathbb{Z}_{S_{2}}\right)$ is also identified with the $W$-invariants of $H_{*}^{T}\left(\mathcal{G} r_{G}, \mathbb{Z}_{S_{2}}\right)$. It remains to show that the $W$-actions on $H_{*}^{T}\left(\mathcal{G} r_{G}, \mathbb{Z}_{S_{2}}\right)$ and $\mathcal{O}\left(B_{e^{T}}^{\vee}\right)$ correspond to each other under the isomorphism $\sigma^{T}$ in Theorem 6.1 (2). For this, it is enough to argue over $\operatorname{Frac}\left(R_{T}\right)$ and use equivariant localization (Remark 3.4) again. The details are left to the reader.

\section{ACKNOWLEDGMENT}

The research of Z.Y. is supported by the National Science Foundation under the agreement No. DMS-0635607. Any opinions, findings and conclusions or recommendations expressed in this material are those of the authors and do not necessarily reflect the views of the National Science Foundation. The research of X.Z. was partially conducted during the period he was employed by the Clay Mathematics Institute as a Liftoff Fellow.

\section{REFERENCES}

[B53] Borel, A. Sur la cohomologie des espaces fibrés principaux et des espaces homogènes de groupes de Lie compacts. Ann. of Math. (2) 57, (1953). 115-207. MR0051508 (14:490e)

[B60] Borel, A. Commutative subgroups and torsion in compact Lie groups. Bull. amer. Math. Soc. 661960 285-288. MR0117299 (22:8080)

[B58] Bott, R. The space of loops on a Lie group. Michigan Math. J. 5(1958), 35-61. MR 0102803 (21:1589)

[G95] Ginzburg, V. Perverse sheaves on a Loop group and Langlands' duality. arXiv:math/9511007. 
[J03] Jantzen, J. C. Representations of algebraic groups. Second edition. Mathematical Surveys and Monographs, 107. American Mathematical Society, Providence, RI, 2003. MR2015057 (2004h:20061)

[K87] Keny, S. V. Existence of regular nilpotent elements in the Lie algebra of a simple algebraic group in bad characteristics. J. Algebra 108 (1987), no. 1, 195-201. MR887203 (88d:17011)

[KK86] Kostant, B; Kumar, S. The nil Hecke ring and the cohomology of $G / P$ for a Kac-Moody group G. Adv. in Math. 62 (1986), 187-237. MR866159 (88b:17025b)

[KNR94] Kumar, S; Narasimhan, M.S; Ramanathan, A. Infinite Grassmannians and moduli spaces of $G$-bundles. Math. Annalen 300 (1994), 41-75. MR.1289830 (96e:14011)

[L81] Lusztig, G. Singularities, character formulas, and a $q$-analog of weight multiplicities. Analysis and topology on singular spaces, II, III (Luminy, 1981), 208-229, Astérisque, 101-102, Soc. Math. France, Paris, 1983. MR737932 (85m:17005)

[M80] Matsumura, H. Commutative algebra. Second edition. Mathematics Lecture Note Series, 56. Benjamin/Cummings Publishing Co., Inc., Reading, Mass., 1980. MR575344 (82i:13003)

[MV07] Mirković, I.; Vilonen, K. Geometric Langlands duality and representations of algebraic groups over commutative rings. Ann. of Math. (2) 166 (2007), no. 1, 95-143. MR2342692 $(2008 \mathrm{~m}: 22027)$

[N06] Ngô, B-C. Fibration de Hitchin et endoscopie. Invent. Math. 164 (2006), no. 2, 399-453. MR:2218781 (2007k:14018)

[PS86] Pressley, A; Segal, G. Loop groups. The Clarendon Press Oxford University Press, New York, 1986. Oxford Science Publications. MR900587 (88i:22049)

[Sa72] Saavedra Rivano, N. Catégories Tannakiennes. Lecture Notes in Mathematics, Vol. 265. Springer-Verlag, Berlin-New York, 1972. MR0338002 (49:2769)

[So00] Sorger, C. Lectures on moduli of principal G-bundles over algebraic curves. School on Algebraic Geometry (Trieste, 1999), 1-57, ICTP Lect. Notes, 1, Abdus Salam Int. Cent. Theoret. Phys., Trieste, 2000. MR:1795860 (2002h:14017)

[Sp66] Springer, T. A. Some arithmetical results on semi-simple Lie algebras. Inst. Hautes Études Sci. Publ. Math. No. 30 (1966), 115-141. MR0206171 (34:5993)

Institute for Advanced Study, Einstein Drive, Princeton, New Jersey 08540

Current address: Department of Mathematics, Massachusetts Institute of Technology,

77 Massachusetts Avenue, Cambridge, Massachusetts 02139

E-mail address: zyun@math.mit.edu

Department of Mathematics, Harvard University, One Oxford Street, Cambridge, MASSACHUSETTS 02138

E-mail address: xinwenz@math.harvard.edu 